\title{
Lectins from red algae and their biomedical potential
}

\author{
Ram Sarup Singh ${ }^{1}$ - Amandeep Kaur Walia ${ }^{1}$
}

Received: 31 July 2017 /Revised and accepted: 6 November 2017 /Published online: 20 November 2017

(C) Springer Science+Business Media B.V., part of Springer Nature 2017

\begin{abstract}
Lectins are unique proteins or glycoproteins of nonimmune origin that bind specifically to carbohydrates. They recognise and interact reversibly to either free carbohydrates or glycoconjugates, without modifying their structure. Lectins are highly diverse and widely distributed in nature and have been extensively reported from various red algae species. Numerous red algae species have been reported to possess lectins having carbohydrate specificity towards complex glycoproteins or high-mannose $\mathrm{N}$-glycans. These lectin-glycan interactions further trigger many biochemical responses which lead to their extensive use as valuable tools in biomedical research. Thus, owing to their exceptional glycan recognition property, red algae lectins are potential candidate for inhibition of various viral diseases. Hence, the present report integrates existing information on the red algae lectins, their carbohydrate specificity, and characteristics of purified lectins. Further, the review also reports the current state of research into their anti-viral activity against various enveloped viruses such as HIV, hepatitis, influenza, encephalitis, coronavirus and herpes simplex virus and other biomedical activities such as anti-cancer, anti-microbial, anti-inflammatory, antinociceptive and acaricidal activities.
\end{abstract}

Keywords Rhodophyceae $\cdot$ Lectins $\cdot$ Haemagglutination . Carbohydrate specificity $\cdot$ Anti-viral

Ram Sarup Singh

rssingh11@lycos.com; rssbt@pbi.ac.in

1 Carbohydrate and Protein Biotechnology Laboratory, Department of Biotechnology, Punjabi University, Patiala, Punjab 147 002, India

\section{Introduction}

Lectins (haemagglutinins) are carbohydrate-binding proteins/ glycoproteins of non-immune origin which agglutinate cells or precipitate glycoconjugates (Dixion, 1981). Lectins bind reversibly to carbohydrates and interact specifically with glycans linked to membrane bound or soluble glycoconjugates without altering their covalent structure (Liener et al. 1986). Various non-covalent forces such as hydrogen bonding, hydrophobic interactions and van der Waal's forces are involved in lectinsugar interactions (Mirelman, 1986). Proteins are considered as lectins if they fulfill the following conditions (Rüdiger and Gabius, 2001): (a) should bind carbohydrates, (b) should not modify the carbohydrates they bind to and (c) should vary from immunoglobulins. Further, the "carbohydrate recognition domain" of lectins resides in their polypeptide sequence which is the underlying basis of carbohydrate-binding property of most lectins, as revealed by their amino acid sequence analysis (Drickamer, 1988). Lectins have potential to agglutinate erythrocytes, lymphocytes and microbial cells based on their carbohydrate specificity (Sharon and Lis 2004). High amounts of sialoglycoproteins are present on the surface of red blood cells (Furthmayr 1977). Agglutination results in cross-linking of several blood cells as lectins interact specifically to terminal sugar residues on the surface of erythrocytes (Khan et al. 2002). Thus, erythrocyte agglutination is a characteristic feature of lectins which plays a role in the determination of lectin activity based on haemagglutination assays (Ambrosi et al. 2005). In nature, lectins are present amongst wide variety of organisms like animals, higher plants, algae, fungi, protozoa, yeast, mushroom, corals, prokaryotes, invertebrates and vertebrates (Singh et al. 1999). Plant lectins have been extensively explored for their various biological applications such as anticancer, immunomodulatory and pro-healing (Teixeira et al. 2012). Apart from them, lectins from microfungi (Singh et al. 
2011), mushrooms (Singh et al. 2010, 2016) and algae (Singh et al. 2015) have also been explored for various biomedical applications. Amongst algae, agglutinins have been most extensively reported from the Rhodophyceae, Chlorophyceae, Cyanophyceae and Phaeophyceae (Akkouh et al. 2015; Singh et al. 2017; Teixeira et al. 2012).

Recently, there has been increased interest in lectins from various marine species such as algae, sponge, mollusk, fish and arthropod, owing to their potential value in various medical applications (Rabelo et al. 2012; Cheung et al. 2015). Marine algae are excellent sources of novel lectin molecules for research and have wide applications in various fields such as pharmaceutical science, medicine, food science, glycobiology and biochemistry (Praseptiangga 2015, 2017). Over the years, marine algae from varied regions such as Britain (Boyd et al. 1966; Blunden et al. 1975), USA (Shiomi et al. 1979), Japan (Shiomi et al. 1980) and Brazil (Ainouz et al. 1992) have been screened for the presence of haemagglutinins. Algal lectins, particularly from red algae, share some common characteristics of monomeric forms, low molecular weight, thermostability and divalent cationindependent hemagglutination, along with affinity only for glycoproteins but not for monosaccharides (Hori et al. 1990; Rogers and Hori, 1993). Thus, algal lectins are molecules with low molecular weight and may be less antigenic when used in biological models as depicted by several studies on biological applications of lectins from algae (Teixeira et al. 2012).

Amongst red marine algae, lectin from Griffithsia sp. (GRFT) has been widely studied (Mori et al. 2005). Owing to high expression level, potency, stability and current indication for topical delivery, GRFT acts as best-case scenario for non-vaccine plant made pharmaceuticals; however, there are many challenges associated with its bulk production (Fuqua et al. 2015). Lectins from various other red marine algae such as Eucheuma serra (Fukuda et al. 2006), Bryothamnion seaforthii and Bryothamnion triquetrum (Pinto et al. 2009), Acrocystis nana (Anam et al. 2017a, 2017b) and Solieria filiformis (Chaves et al. 2017) have anti-cancer potential and also used as drug delivery agents owing to their carbohydrate specificity. Thus, studies conducted on various algal lectins bring in a new element of research in biomedical applications.

Even though there has been increasing interest towards algal lectins, still limited information is available on its structural characteristics. Thus, the current review compiles lectins particularly from red algae along with their haemagglutination activity and carbohydrate specificity. It also focuses on physiochemical characteristics of lectins from red algae along with their structural studies and their biomedical potential against various diseases.

\section{Haemagglutination activity of lectins from red algae}

Agglutination is a remarkable property of lectins which occurs owing to polyvalent nature of protein along with its affinity towards specific carbohydrates on cell surface. Based on their ability to agglutinate human blood type erythrocytes, lectins are classified as specific or non-specific (Sharon and Lis 1972). Agglutination preferences of crude lectins and lectins purified from various red algae species are tabulated in Tables 1 and 2, respectively. Around 800 algal species have been analysed for the presence of lectins and approximately $60 \%$ of analysed species showed haemagglutination activity (Teixeira et al. 2012). Crude lectin extract from Porphyra sp. specifically agglutinates human blood type B erythrocytes only (De Souza et al. 2007). Crude lectins from Acanthophora spicifera (Ainouz et al. 1992), Bossiella cretacea, Nemalion vermiculare, Rhodomela munita and Tichocarpus crinitus (Chernikov et al. 2007) specifically agglutinate human blood type $\mathrm{O}$ erythrocytes. Purified lectin from Vidalia obtusiloba preferentially agglutinates human blood type $\mathrm{O}$ erythrocytes followed by A erythrocytes (Melo et al. 2004), whereas Enantiocladia duperreyi purified lectin exhibits slight preference for bromelain-treated human blood type $\mathrm{O}$ erythrocytes followed by B and A erythrocytes (Benevides et al. 1998). Amansia multifida (Ainouz et al. 1992) crude lectin specifically agglutinates human blood type $\mathrm{O}$ and $\mathrm{A}$ erythrocytes, whereas Gracilaria tikvahiae G-3, Gracilaria verrucosa G16S and Eucheuma nudum crude lectins agglutinate human blood type A and B erythrocytes only (Chiles and Bird 1989).

Crude lectins from Gracilaria ferox and Hypnea musciformis exhibit non-specific agglutination of human erythrocytes (Ainouz and Sampaio 1991). Purified lectin from Ptilota serrata is non-specific towards human blood groups and agglutinates all native and enzyme (papain)-treated erythrocytes to similar extent (Sampaio et al. 1999). Human red blood cell membrane sialoglycoprotein (glycophorin) consists of 15-O-glycosidically linked chains attached to the sialylated T-antigen structure (Marchesi et al. 1976) along with one biantennary complex carbohydrate chain which is $\mathrm{N}$-glycosidically linked to asparagines (Yoshima et al. 1980). Tichocarpus crinitus purified lectin agglutinates various erythrocytes such as human $\mathrm{ABO}$ and rat erythrocytes but has highest agglutination titre with rabbit erythrocytes (Molchanova et al. 2010). Human erythrocytes possess only Neu5Ac sialic acid linked to glycoproteins (Reuter et al. 1980), whereas rabbit erythrocytes express high concentration of Neu5Ac and Neu5,9 $\mathrm{Ac}_{2}$ along with Neu9Ac5Gc linked to glycoprotein on their cell surface (Pfeil et al. 1980).

Most of the red algal lectins preferentially agglutinate rabbit and sheep erythrocytes. Acanthophora spicifera crude lectin exhibits haemagglutination activity with only rabbit erythrocytes (Chiles and Bird 1989). Crude lectin extracts from Agardhiella ramosissima, Halymenia agardhii and Halymenia floresia also exhibit specific agglutination towards rabbit and sheep erythrocytes (Chiles and Bird 1989). Crude lectins from Agardhiella tenera, E. nudum, Gymnogongrus griffithsiae, G. tikvahiae, G. verrucosa, H. agardhii, H. floresi and 
Table 1 Biological action spectrum of crude lectins from red algae

Red algae Haemagglutination activity with erythrocyte type/s

Acanthophora spicifera

A. spicifera

A. spicifera

Acrocystis nana

Agardhiella ramosissima

A. tenera

Amansia multifida

Bossiella cretacea

Bryothamnion seaforthii

B. triquetrum

Callophyllis crispata

C. japonica

Campylaephora crassa

Carpopeltis flabellata

Chondria crassicaulis

Chondrus ocellatus

Chrysymenia wrightii

Cryptonemia crenulata

Dictyurus occidentalis

Enantiocladia duperreyi

E. denticulatum

E. nudum

E. nudum

Galaxaura fastigiata

G. filamentosa

Gelidiela acerosa

Gelidiopsis gracilis

Gelidium amansii

G. coarctatum

G. divaricatum

Georgiella confluens

Gigartina skottsbergii

Gloiopeltis furcata

G. furcata

Gracilaria bursa-pastoris

G. bailiniae

G. bangmeiana

G. bursa-pastoris

G. cearensis

G. cervicornis

G. changii
Rabbit $^{\mathrm{a}, \mathrm{b}, \mathrm{c}, \mathrm{d}}$, goat ${ }^{\mathrm{b}}$, chicken ${ }^{\mathrm{a}, \mathrm{d}}$, pig $^{\mathrm{b}, \mathrm{c}}$ and human $\mathrm{O}^{\mathrm{b}, \mathrm{d}}$ erythrocytes

Rabbit erythrocytes

Rabbit $^{\mathrm{a}, \mathrm{b}}$, sheep ${ }^{\mathrm{a}, \mathrm{b}}$ and chicken ${ }^{\mathrm{a}, \mathrm{b}}$ erythrocytes

Rabbit ${ }^{\text {a }}$ erythrocytes

Rabbit and sheep erythrocytes

Rabbit and sheep erythrocytes

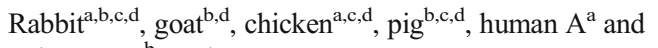
human $\mathrm{O}^{\mathrm{b}}$ erythrocytes

Human $\mathrm{O}$ erythrocytes

Rabbit $^{\mathrm{a}}$, chicken ${ }^{\mathrm{a}}$ and cow $^{\mathrm{a}}$ erythrocytes

Rabbit ${ }^{\mathrm{a}, \mathrm{b}, \mathrm{c}, \mathrm{d}, \mathrm{e}}$, goat ${ }^{\mathrm{b}, \mathrm{c}, \mathrm{d}}$, pig ${ }^{\mathrm{a}, \mathrm{b}, \mathrm{c}, \mathrm{d}, \mathrm{e}}$, human $\mathrm{A}^{\mathrm{b}, \mathrm{c}, \mathrm{d}}$, human $\mathrm{B}^{\mathrm{a}, \mathrm{b}, \mathrm{c}, \mathrm{d}}$, human $\mathrm{O}^{\mathrm{a}, \mathrm{b}, \mathrm{c}, \mathrm{d}, \mathrm{e}}$ and human $\mathrm{AB}^{\mathrm{b}, \mathrm{c}, \mathrm{d}}$ erythrocytes

Horse $^{\mathrm{a}, \mathrm{e}, \mathrm{f}}$ erythrocytes

Sheep ${ }^{\mathrm{a}, \mathrm{e}, \mathrm{f}}$ erythrocytes

Sheep ${ }^{\text {a,f }}$ erythrocytes

Rabbit ${ }^{\mathrm{a}}$ erythrocytes

Sheep ${ }^{\mathrm{a}, \mathrm{f}}$ erythrocytes

Sheep ${ }^{\mathrm{a}, \mathrm{f}}$ erythrocytes

Sheep ${ }^{\mathrm{a}, \mathrm{f}}$ erythrocytes

Rabbit ${ }^{\mathrm{a}}$ erythrocytes

Rabbit ${ }^{\mathrm{a}, \mathrm{b}, \mathrm{c}, \mathrm{d}}$ erythrocytes

Rabbitc ${ }^{\mathrm{c}}$, human $\mathrm{A}^{\mathrm{b}, \mathrm{c}, \mathrm{d}}$, human $\mathrm{B}^{\mathrm{b}, \mathrm{c}, \mathrm{d}}$, human $\mathrm{O}^{\mathrm{a}, \mathrm{b}, \mathrm{c}, \mathrm{d}, \mathrm{e}}$ and human $\mathrm{AB}^{\mathrm{b}, \mathrm{c}, \mathrm{d}}$ erythrocytes

Rabbit ${ }^{\mathrm{a}, \mathrm{b}}$ and sheep ${ }^{\mathrm{a}, \mathrm{b}, \mathrm{e}}$ erythrocytes

Rabbit, sheep, human A and human B erythrocytes

Rabbit and sheep erythrocytes

Sheep ${ }^{\mathrm{a}, \mathrm{f}}$ erythrocytes

Rabbit $^{\mathrm{a}, \mathrm{b}}$, sheep ${ }^{\mathrm{a}, \mathrm{b}}$ and chicken ${ }^{\mathrm{a}, \mathrm{b}}$ erythrocytes

Goat $^{\mathrm{c}, \mathrm{d}, \mathrm{e}}$ and chicken ${ }^{\mathrm{a}, \mathrm{b}, \mathrm{c}, \mathrm{e}}$ erythrocytes

Rabbit ${ }^{\mathrm{a}}$ erythrocytes

Sheep ${ }^{\mathrm{a}, \mathrm{f}}$ erythrocytes

Rabbit $^{\mathrm{d}}$ erythrocytes

Rabbit erythrocytes

Chicken $^{\mathrm{a}, \mathrm{b}, \mathrm{c}, \mathrm{d}, \mathrm{e}}$ and sheep $\mathrm{p}^{\mathrm{a}, \mathrm{b}, \mathrm{c}, \mathrm{d}, \mathrm{e}}$ erythrocytes

Rabbit $^{\mathrm{a}}$, chicken ${ }^{\mathrm{d}}$, goat ${ }^{\mathrm{b}, \mathrm{c}, \mathrm{d}}$, sheep ${ }^{\mathrm{a}, \mathrm{b}, \mathrm{c}, \mathrm{d}, \mathrm{e}}$, human $\mathrm{A}^{\mathrm{a}, \mathrm{b}, \mathrm{c}, \mathrm{d}}$, human $\mathrm{B}^{\mathrm{b}, \mathrm{d}}$ and human $\mathrm{O}^{\mathrm{a}, \mathrm{c}, \mathrm{d}}$ erythrocytes

Rabbit ${ }^{\mathrm{a}}$, sheep ${ }^{\mathrm{a}, \mathrm{e}, \mathrm{f}}$, human $\mathrm{A}^{\mathrm{e}}$, human $\mathrm{B}^{\mathrm{e}}$ and human $\mathrm{O}^{\mathrm{e}}$ erythrocytes

Rabbit, sheep, chicken, human A, human B and human AB erythrocytes

Rabbit $^{\mathrm{a}, \mathrm{e}, \mathrm{f}}$, sheep ${ }^{\mathrm{a}, \mathrm{e}, \mathrm{f}}$, horse ${ }^{\mathrm{f}}$, chicken $^{\mathrm{a}, \mathrm{f}}$ and human $\mathrm{A}^{\mathrm{f}}$ erythrocytes

Rabbit $^{\mathrm{a}, \mathrm{b}, \mathrm{e}}$, sheep ${ }^{\mathrm{b}}$ and chicken ${ }^{\mathrm{a}, \mathrm{b}, \mathrm{e}}$ erythrocytes

Chicken $^{\mathrm{a}, \mathrm{b}}$ erythrocytes

Rabbit ${ }^{\mathrm{a}}$ erythrocytes

Rabbit ${ }^{\mathrm{a}}$ erythrocytes

Rabbit $^{\mathrm{a}}$ and chicken ${ }^{\mathrm{a}}$ erythrocytes

Sheep ${ }^{\mathrm{a}, \mathrm{b}}$ and chicken ${ }^{\mathrm{b}}$ erythrocytes
Reference(s)

Ainouz et al. (1992)

Chiles and Bird (1989)

Dinh et al. (2009)

Anam et al. (2017a)

Chiles and Bird (1989), Bird et al. (1993)

Bird et al. (1993)

Ainouz et al. (1992)

Chernikov et al. (2007)

Ainouz and Sampaio (1991)

Ainouz et al. (1992)

Hori et al. (1988b)

Hori et al. (1988b)

Hori et al. (1988b)

Hori et al. (1986a)

Hori et al. (1988b)

Hori et al. (1988b)

Hori et al. (1988b)

Ainouz and Sampaio (1991)

Ainouz et al. (1992)

Ainouz et al. (1992)

Dinh et al. (2009)

Chiles and Bird (1989)

Bird et al. (1993)

Hori et al. (1988b)

Dinh et al. (2009)

Ainouz et al. (1992)

Ainouz and Sampaio (1991)

Hori et al. (1988b)

Ainouz et al. (1992)

Zheng and Lai-Sheng (2002)

De Souza et al. (2007)

De Souza et al. (2007)

Hori et al. (1988b)

Zheng and Lai-Sheng (2002)

Hori et al. (1988b)

Dinh et al. (2009)

Dinh et al. (2009)

Hori et al. (1986a)

Ainouz and Sampaio (1991)

Ainouz and Sampaio (1991)

Dinh et al. (2009) 
Table 1 (continued)

\begin{tabular}{|c|c|c|}
\hline Red algae & Haemagglutination activity with erythrocyte type/s & Reference(s) \\
\hline G. cornea & Chicken ${ }^{\mathrm{a}}$ erythrocytes & $\begin{array}{l}\text { Ainouz and Sampaio } \\
\text { (1991), Lima et al. (2005) }\end{array}$ \\
\hline G. eucheumatoides & $\begin{array}{l}\text { Rabbit }^{\mathrm{a}, \mathrm{b}} \text {, sheep } \\
\text { and human } \mathrm{O}^{\mathrm{a}, \mathrm{b}, \mathrm{e}} \text { erythrocytes }\end{array}$ & Dinh et al. (2009) \\
\hline G. ferox & $\begin{array}{l}\text { Sheep }{ }^{\mathrm{a}} \text {, rabbit }{ }^{\mathrm{a}} \text {, chicken }{ }^{\mathrm{a}} \text {, } \text { cow }^{\mathrm{a}} \text {, human } \mathrm{A}^{\mathrm{a}} \text {, human } \mathrm{B}^{\mathrm{a}} \text {, } \\
\text { human } \mathrm{AB}^{\mathrm{a}} \text { and human } \mathrm{O}^{\mathrm{a}} \text { erythrocytes }\end{array}$ & Ainouz and Sampaio (1991) \\
\hline G. fisheri & Rabbit, sheep and goose erythrocytes & Boonsri et al. (2017) \\
\hline G. salicornia & Rabbit $^{\mathrm{a}, \mathrm{b}, \mathrm{e}}$, sheep ${ }^{\mathrm{a}, \mathrm{b}, \mathrm{e}}$ and chicken ${ }^{\mathrm{a}, \mathrm{b}, \mathrm{e}}$ erythrocytes & Dinh et al. (2009) \\
\hline G. sjoestedtii & Sheep $^{\mathrm{a}}$ and chicken ${ }^{\mathrm{a}}$ erythrocytes & Ainouz and Sampaio (1991) \\
\hline G. textorii & $\begin{array}{l}\text { Rabbit }^{\mathrm{a}, \mathrm{e}, \mathrm{f}} \text {, sheep } \\
\text { and human } \mathrm{O}^{\mathrm{a}, \mathrm{e}, \mathrm{f}} \text {, horse } \text { erythrocytes }^{\mathrm{a}, \mathrm{e}, \mathrm{f}} \text {, human } \mathrm{A}^{\mathrm{a}, \mathrm{f}} \text {, human } \mathrm{B}^{\mathrm{a}, \mathrm{f}} \\
\end{array}$ & Hori et al. (1988b) \\
\hline G. tikvahiae & Rabbit and sheep erythrocytes & Bird et al. (1993) \\
\hline G. tikvahiae & Rabbit, sheep, human A and human B erythrocytes & Chiles and Bird (1989) \\
\hline G. verrucosa & $\begin{array}{l}\text { Rabbitt }^{\mathrm{f}} \text {, sheep } \\
\text { human } \mathrm{B}^{\mathrm{a}, \mathrm{f}} \text {, horse } \\
\text { and human } \mathrm{O}^{\mathrm{a}, \mathrm{e}, \mathrm{f}} \text { erythrocytes }\end{array}$ & Hori et al. (1988b) \\
\hline G. verrucosa & $\begin{array}{l}\text { Horse, cow, sheep, rabbit, guinea pig, carp and chicken } \\
\text { erythrocytes }\end{array}$ & Shiomi et al. (1981) \\
\hline G. verrucosa & Rabbit and sheep erythrocytes & Bird et al. (1993) \\
\hline G. verrucosa & Rabbit, sheep, human A and human B erythrocytes & Chiles and Bird (1989) \\
\hline Gracilariopsis lemaneiformis & Sheep erythrocytes & Bird et al. (1993) \\
\hline Grateloupia filicina & Sheep ${ }^{\mathrm{a}, \mathrm{e}, \mathrm{f}}$ erythrocytes & Hori et al. (1988b) \\
\hline G. filicina & Rabbit erythrocytes & Zheng and Lai-Sheng (2002) \\
\hline G. filicina & $\begin{array}{l}\text { Rabbit }^{\mathrm{a}, \mathrm{b}} \text {, sheep }{ }^{\mathrm{a}, \mathrm{b}} \text {, chicken } \\
\text { and human } \mathrm{O}^{\mathrm{a}, \mathrm{b}} \text { erythrocytes }\end{array}$ & Dinh et al. (2009) \\
\hline Gymnogongrus flabelliformis & Sheep ${ }^{\mathrm{a}, \mathrm{f}}$ erythrocytes & Hori et al. (1988b) \\
\hline G. griffithsiae & Rabbit and sheep erythrocytes & Bird et al. (1993) \\
\hline G. turquetti & Chicken $^{\mathrm{d}}$ and sheep ${ }^{\mathrm{d}}$ erythrocytes & De Souza et al. (2007) \\
\hline Halymenia sp. & Rabbit $^{\mathrm{a}}$ and human $\mathrm{B}^{\mathrm{a}, \mathrm{e}}$ erythrocytes & De Souza et al. (2007) \\
\hline H. agardhii & Rabbit and sheep erythrocytes & $\begin{array}{l}\text { Chiles and Bird (1989), } \\
\text { Bird et al. (1993) }\end{array}$ \\
\hline H. floresia & Rabbit and sheep erythrocytes & $\begin{array}{l}\text { Chiles and Bird (1989), } \\
\text { Bird et al. (1993) }\end{array}$ \\
\hline H. maculata & $\begin{array}{l}\text { Rabbit }^{\mathrm{a}, \mathrm{b}, \mathrm{e}} \text {, sheep } \\
\text { and human } \mathrm{O}^{\mathrm{a}, \mathrm{b}, \mathrm{b}} \text { erythrocytes }\end{array}$ & Dinh et al. (2009) \\
\hline Hypnea boergesenii & Rabbit $^{\mathrm{a}, \mathrm{b}, \mathrm{e}}$, sheep ${ }^{\mathrm{a}, \mathrm{b}}$ and chicken ${ }^{\mathrm{a}, \mathrm{b}}$ erythrocytes & Dinh et al. (2009) \\
\hline H. cervicornis & Rabbit $^{\mathrm{a}}$ and cow ${ }^{\mathrm{a}}$ erythrocytes & Ainouz and Sampaio (1991) \\
\hline H. japonica & Rabbit ${ }^{\mathrm{a}}$ erythrocytes & Hori et al. (1986a, 2000) \\
\hline H. japonica & $\begin{array}{l}\text { Rabbit }^{\mathrm{a}, \mathrm{e}, \mathrm{f}} \text {, sheep } \\
\text { and human } \mathrm{O}^{\mathrm{a}, \mathrm{e}, \mathrm{e}, \mathrm{f}} \text {, horse } \text { erythrocytes }^{\mathrm{a}, \mathrm{e}, \mathrm{f}} \text {, human } \mathrm{A}^{\mathrm{a}, \mathrm{f}} \text {, human } \mathrm{B}^{\mathrm{a}, \mathrm{e}, \mathrm{f}} \\
\end{array}$ & Hori et al. (1988b) \\
\hline H. musciformis & $\begin{array}{l}\text { Sheep }{ }^{\mathrm{a}} \text {, rabbit }{ }^{\mathrm{a}} \text {, cow }{ }^{\mathrm{a}} \text {, human } \mathrm{A}^{\mathrm{a}} \text {, human } \mathrm{B}^{\mathrm{a}} \text {, human } \mathrm{AB}^{\mathrm{a}} \\
\text { and human } \mathrm{O}^{\mathrm{a}} \text { erythrocytes }\end{array}$ & Ainouz and Sampaio (1991) \\
\hline H. musciformis & Rabbit and sheep erythrocytes & Bird et al. (1993) \\
\hline H. musciformis & Rabbit erythrocytes & Melo et al. (1997) \\
\hline H. nidulans & $\begin{array}{l}\text { Rabbit }{ }^{\mathrm{a}, \mathrm{b}} \text {, sheep } \mathrm{p}^{\mathrm{a}, \mathrm{b}} \text {, human } \mathrm{A}^{\mathrm{a}, \mathrm{b}} \text {, human } \mathrm{B}^{\mathrm{a}, \mathrm{b}} \text { and } \\
\text { human } \mathrm{O}^{\mathrm{a}, \mathrm{b}} \text { erythrocytes }\end{array}$ & Dinh et al. (2009) \\
\hline H. valentiae & $\begin{array}{l}\text { Rabbit }{ }^{\mathrm{a}, \mathrm{b}} \text {, sheep }{ }^{\mathrm{a}, \mathrm{b}} \text {, human } \mathrm{A}^{\mathrm{b}} \text {, human } \mathrm{B}^{\mathrm{b}} \text { and } \\
\text { human } \mathrm{O}^{\mathrm{b}} \text { erythrocytes }\end{array}$ & Dinh et al. (2009) \\
\hline Kappaphycus alvarezii & Rabbit $^{\mathrm{a}, \mathrm{b}, \mathrm{e}}$ and sheep $\mathrm{p}^{\mathrm{a}, \mathrm{b}}$ erythrocytes & $\begin{array}{l}\text { Dinh et al. (2009), } \\
\text { Le et al. (2009) }\end{array}$ \\
\hline K. alvarezii & Rabbit ${ }^{\mathrm{a}}$ erythrocytes & $\begin{array}{l}\text { Hung et al. (2009), } \\
\text { Hirayama et al. (2016) }\end{array}$ \\
\hline K. striatum & Rabbit $^{\mathrm{a}, \mathrm{b}, \mathrm{e}}$ and sheep ${ }^{\mathrm{a}, \mathrm{b}, \mathrm{e}}$ erythrocytes & $\begin{array}{l}\text { Dinh et al. (2009), } \\
\text { Hung et al. (2011) }\end{array}$ \\
\hline
\end{tabular}


Table 1 (continued)

\begin{tabular}{|c|c|c|}
\hline Red algae & Haemagglutination activity with erythrocyte type/s & Reference(s) \\
\hline Laurencia dichotoma & Rabbit ${ }^{\mathrm{a}}$ erythrocytes & Ainouz et al. (1992) \\
\hline L. microcladia & Rabbit ${ }^{\mathrm{a}, \mathrm{c}}$ erythrocytes & Ainouz et al. (1992) \\
\hline L. obtusa & Rabbit $^{\mathrm{a}, \mathrm{b}}$ and chicken ${ }^{\mathrm{b}}$ erythrocytes & Dinh et al. (2009) \\
\hline L. undulata & $\begin{array}{l}\text { Rabbit }{ }^{\mathrm{a}, \mathrm{e}, \mathrm{f}} \text {, sheep } \\
\text { and human } \mathrm{O}^{\mathrm{e}, \mathrm{e}} \text { erythrocytes }\end{array}$ & Hori et al. (1988b) \\
\hline Meristiella echinocarpa & Rabbit $^{\mathrm{a}, \mathrm{b}, \mathrm{c}, \mathrm{d}}$, goat $\mathrm{g}^{\mathrm{b}, \mathrm{d}}$, chicken ${ }^{\mathrm{a}, \mathrm{b}, \mathrm{d}}$ and pig $^{\mathrm{b}, \mathrm{c}, \mathrm{d}}$ erythrocytes & Ainouz et al. (1992) \\
\hline Nemalion vermiculare & Human $\mathrm{O}$ erythrocytes & Chernikov et al. (2007) \\
\hline Palmaria decipiens & $\begin{array}{l}\text { Rabbit }{ }^{\mathrm{a}, \mathrm{b}, \mathrm{c}, \mathrm{d}, \mathrm{e}}, \text { chicken } \\
\text { human } \mathrm{A}^{\mathrm{a}, \mathrm{b}, \mathrm{b}, \mathrm{c}, \mathrm{c}, \mathrm{d}}, \text {, goat } \\
\text {, human } \mathrm{B}^{\mathrm{b}, \mathrm{d}} \text { and human } \mathrm{O}^{\mathrm{a}, \mathrm{b}, \mathrm{c}, \mathrm{d}} \\
\text { erythrocytes }\end{array}$ & De Souza et al. (2007) \\
\hline P. palmata & $\begin{array}{l}\text { Rabbit }{ }^{\mathrm{a}, \mathrm{b}, \mathrm{e}, \mathrm{f}}, \text { horse } \\
\text { and human } \mathrm{O}^{\mathrm{a}, \mathrm{b}, \mathrm{e}, \mathrm{f}} \text { erythrocytes }\end{array}$ & Kamiya et al. (1982) \\
\hline Pantoneura plocamioides & Rabbit $^{\mathrm{b}, \mathrm{c}}$ and chicken ${ }^{\mathrm{b}, \mathrm{c}}$ erythrocytes & De Souza et al. (2007) \\
\hline Polysiphonia sp. & Rabbit $^{\mathrm{a}}$ and sheep ${ }^{\mathrm{a}, \mathrm{e}, \mathrm{f}}$ erythrocytes & Hori et al. (1988b) \\
\hline Porphyra sp. & Human B erythrocytes & De Souza et al. (2007) \\
\hline Rhodomela munita & Human O erythrocytes & Chernikov et al. (2007) \\
\hline R. subfusca & Sheep ${ }^{\mathrm{a}, \mathrm{f}}$, human $\mathrm{A}^{\mathrm{f}}$ and human $\mathrm{B}^{\mathrm{f}}$ erythrocytes & Hori et al. (1988b) \\
\hline Solieria filiformis & Rabbit $^{\mathrm{a}, \mathrm{b}, \mathrm{c}, \mathrm{d}}$, goat ${ }^{\mathrm{b}, \mathrm{d}}$, chicken ${ }^{\mathrm{b}, \mathrm{c}, \mathrm{d}}$ and pig ${ }^{\mathrm{b}, \mathrm{c}, \mathrm{d}}$ erythrocytes & Ainouz et al. (1992) \\
\hline S. robusta & $\begin{array}{l}\text { Rabbit }^{\mathrm{a}, \mathrm{e}, \mathrm{f}} \text {, sheep } \\
\text { erythrocytes }\end{array}$ & Hori et al. (1988b) \\
\hline Tichocarpus crinitus & Human $\mathrm{O}$ erythrocytes & Chernikov et al. (2007) \\
\hline Vidalia obtusiloba & Rabbit ${ }^{\mathrm{b}, \mathrm{c}}$ and human $\mathrm{A}^{\mathrm{a}}$ erythrocytes & Ainouz et al. (1992) \\
\hline V. volubilis & Rabbit $^{\mathrm{d}}$ erythrocytes & Ainouz et al. (1992) \\
\hline
\end{tabular}

${ }^{a}$ Trypsin-treated erythrocytes

${ }^{\mathrm{b}}$ Papain-treated erythrocytes

${ }^{\mathrm{c}}$ Bromelain-treated erythrocytes

${ }^{\mathrm{d}}$ Subtilisin-treated erythrocytes

${ }^{\mathrm{e}}$ Native erythrocytes

${ }^{\mathrm{f}}$ Pronase-treated erythrocytes

${ }^{\mathrm{g}}$ Neuraminidase-treated erythrocytes

H. musciformis specifically agglutinate rabbit and sheep erythrocytes, whereas Gracilariopsis lamaneiformis crude lectin agglutinates only sheep erythrocytes (Bird et al. 1993). Purified lectin from red algae $A$. tenera (abundant on northeast coast of the USA) exhibits high activity with guinea pig and rabbit erythrocytes as compared to other erythrocytes (Shiomi et al. 1979). Gelidium divaricatum and Grateloupia filicina crude lectin extracts exhibit agglutination only with rabbit erythrocytes (Zheng and Lai-Sheng 2002). Crude protein extract from Gracilaria fisheri (GPE) strongly agglutinates rabbit erythrocytes with agglutination titre of 1:512 followed by sheep and goose erythrocytes with haemagglutination titre of 1:128 and 1:64, respectively (Boonsri et al. 2017). However, GPE is unable to agglutinate rat, mouse and hamster erythrocytes (Boonsri et al. 2017).

A lectin purified from Aglaothamnion callophyllidicola specifically agglutinates horse erythrocytes (Shim et al. 2012). Callophyllis crispata crude lectin agglutinates only native and enzyme-treated horse erythrocytes (Hori et al. 1988b). Low molecular weight $G$. verrucosa haemagglutinin (L-GVH) has low haemagglutination activity than high molecular weight G. verrucosa haemagglutinin (H-GVH) but strongly agglutinates horse and rabbit erythrocytes as compared to other erythrocytes (Kakita et al. 1999). Serraticardia maxima purified lectin agglutinates non-treated horse erythrocytes more strongly as compared to other animal erythrocytes (Shiomi et al. 1980). However, purified lectin from A. tenera and C. purpureum exhibits weak activity towards horse erythrocytes (Shiomi et al. 1979; Kamiya et al. 1980). Pig, sheep and horse erythrocytes consist of high proportion of Neu5Gc as parent sialic acid (Cabezas 1973) along with traces of Neu5Ac in pig and horse erythrocytes (Cabezas and Cabezas 1973).

Gracilaria verrucosa purified lectin exhibited high agglutination activity with chicken erythrocytes as compared to various other red algae agglutinins (Shiomi et al. 1981). Gracilaria cornea crude lectin agglutinates trypsin-treated chicken 
Table 2 Biological action spectrum of purified lectins from red algae

\begin{tabular}{|c|c|c|}
\hline Red algae & Haemagglutination activity with erythrocyte type/s & Reference(s) \\
\hline Agardhiella tenera & $\begin{array}{l}\text { Guinea pig, rabbit, mouse, horse, sheep and human A, B } \\
\text { and O erythrocytes }\end{array}$ & Shiomi et al. (1979) \\
\hline Aglaothamnion callophyllidicola & Horse erythrocytes & Shim et al. (2012) \\
\hline Bryothamnion triquetrum & Rabbit ${ }^{\mathrm{a}, \mathrm{b}}$ erythrocytes & Nascimento et al. (2015) \\
\hline Carpopeltis flabellata & Rabbit $^{\mathrm{a}}$ erythrocytes & $\begin{array}{l}\text { Hori et al. (1986a), } \\
\quad \text { Matsubara et al. (1996) }\end{array}$ \\
\hline C. flabellata & Rabbit $^{\mathrm{a}, \mathrm{e}}$, mouse $^{\mathrm{a}, \mathrm{e}}$, horse $^{\mathrm{a}, \mathrm{e}}$, chicken $^{\mathrm{a}}$ and human ${ }^{\mathrm{e}}$ erythrocytes & Hori et al. (1987) \\
\hline Cystoclonium purpureum & $\begin{array}{l}\text { Rabbit, guinea pig, mouse, horse, human A, human B } \\
\text { and human O erythrocytes }\end{array}$ & Kamiya et al. (1980) \\
\hline Enantiocladia duperreyi & Human $\mathrm{A}^{\mathrm{c}}$, human $\mathrm{B}^{\mathrm{c}}$ and human $\mathrm{O}^{\mathrm{c}}$ erythrocytes & Benevides et al. (1998) \\
\hline Eucheuma amakusaensis & Sheep $^{\mathrm{a}, \mathrm{e}}$ and rabbit ${ }^{\mathrm{a}}$ erythrocytes & $\begin{array}{l}\text { Kawakubo et al. (1997), } \\
\text { Kawakubo et al. (1999) }\end{array}$ \\
\hline E. cottonii & Sheep ${ }^{\mathrm{a}, \mathrm{e}}$ and rabbit ${ }^{\mathrm{a}}$ erythrocytes & Kawakubo et al. $(1997,1999)$ \\
\hline Georgiella confluens & Chicken $^{\mathrm{a}, \mathrm{e}}$ erythrocytes & Souza et al. (2010) \\
\hline Gracilaria ornata & Rabbit $^{\mathrm{a}}$ and chicken ${ }^{\mathrm{a}}$ erythrocytes & Leite et al. (2005) \\
\hline G. tikvahiae & Rabbit, sheep, human A and human B erythrocytes & Chiles and Bird (1990) \\
\hline G. verrucosa & $\begin{array}{l}\text { Horse, cow, sheep, rabbit, guinea pig, carp and chicken } \\
\text { erythrocytes }\end{array}$ & Shiomi et al. (1981) \\
\hline G. verrucosa & Rabbit, guinea pig and sheep ${ }^{\mathrm{f}}$ erythrocytes & Kakita et al. (1997) \\
\hline G. verrucosa & Horse, rabbit and sheep ${ }^{f}$ erythrocytes & Kakita et al. (1999) \\
\hline Hypnea cervicornis & Rabbit ${ }^{a}$ erythrocytes & Nascimento et al. (2006) \\
\hline H. japonica & Rabbit ${ }^{\mathrm{a}}$ erythrocytes & $\begin{array}{l}\text { Matsubara et al. (1996), } \\
\text { Hori et al. (2000) }\end{array}$ \\
\hline H. japonica & $\begin{array}{l}\text { Rabbit, horse, sheep, chicken and human A, human B } \\
\text { and human O erythrocytes }\end{array}$ & Hori et al. (1986b) \\
\hline H. musciformis & $\begin{array}{l}\text { Rabbit }^{\mathrm{a}, \mathrm{b}, \mathrm{c}, \mathrm{d}, \mathrm{e}} \text {, } \operatorname{cow}^{\mathrm{a}}, \text { sheep } \\
\text { and human } \mathrm{O}^{\mathrm{a}, \mathrm{b}, \mathrm{c}, \mathrm{d}, \mathrm{e}, \mathrm{e}} \text { erythrocytes }\end{array}$ & Nagano et al. (2002) \\
\hline Pterocladiella capillacea & Rabbit $^{\mathrm{a}, \mathrm{c}, \mathrm{d}}$ erythrocytes & Oliveira et al. (2002) \\
\hline P. capillacea & Rabbit ${ }^{\mathrm{a}}$ erythrocytes & Silva et al. (2010) \\
\hline Ptilota filicina & Human $\mathrm{A}^{\mathrm{b}, \mathrm{e}}$, human $\mathrm{B}^{\mathrm{b}, \mathrm{e}}$ and human $\mathrm{O}^{\mathrm{b}, \mathrm{e}}$ erythrocytes & Sampaio et al. (1998) \\
\hline P. plumosa & Human $\mathrm{A}^{\mathrm{b}}$, human $\mathrm{B}^{\mathrm{b}, \mathrm{e}}$ and human $\mathrm{O}^{\mathrm{b}}$ erythrocytes & Sampaio et al. (2002) \\
\hline P. serrata & Human $\mathrm{A}^{\mathrm{b}, \mathrm{e}}$, human $\mathrm{B}^{\mathrm{b}, \mathrm{e}}$ and human $\mathrm{O}^{\mathrm{b}, \mathrm{e}}$ erythrocytes & Sampaio et al. (1999) \\
\hline Serraticardia maxima & $\begin{array}{l}\text { Horse }^{\mathrm{a}, \mathrm{b}, \mathrm{e}}, \operatorname{cow}^{\mathrm{a}, \mathrm{b}, \mathrm{e}}, \text { sheep }^{\mathrm{a}, \mathrm{b}, \mathrm{e}}, \text { rabbit }^{\mathrm{a}, \mathrm{b}, \mathrm{e}} \text {, guinea pig }{ }^{\mathrm{a}, \mathrm{b}, \mathrm{e}} \\
\text { mouse }^{\mathrm{a}, \mathrm{b}, \mathrm{e}} \text { and chicken } \\
\text { a,b,e }\end{array}$ & Shiomi et al. (1980) \\
\hline Solieria filiformis & Rabbit $^{\text {a erythrocytes }}$ & Chaves et al. (2017) \\
\hline S. chordalis & Human $\mathrm{A}^{\mathrm{b}, \mathrm{c}, \mathrm{e}, \mathrm{g}}$, human $\mathrm{B}^{\mathrm{b}, \mathrm{c}, \mathrm{e}, \mathrm{g}}$ and human $\mathrm{O}^{\mathrm{b}, \mathrm{c}, \mathrm{e}, \mathrm{g}}$ erythrocytes & Rogers and Toplis (1983) \\
\hline S. robusta & Rabbit ${ }^{\mathrm{a}, \mathrm{e}, \mathrm{f}}$ erythrocytes and human $\mathrm{A}^{\mathrm{a}}$ erythrocytes & Hori et al. (1988a) \\
\hline S. robusta & Rabbit $^{\mathrm{a}}$ erythrocytes & Matsubara et al. (1996) \\
\hline Tichocarpus crinitus & Rabbit, rat, human A, human B and human $\mathrm{O}$ erythrocytes & Molchanova et al. (2010) \\
\hline Vidalia obtusiloba & Rabbit ${ }^{\mathrm{c}}$, human $\mathrm{O}^{\mathrm{c}}$ and human $\mathrm{A}^{\mathrm{c}}$ erythrocytes & Melo et al. (2004) \\
\hline
\end{tabular}

\footnotetext{
${ }^{\text {a }}$ Trypsin-treated erythrocytes

${ }^{\mathrm{b}}$ Papain-treated erythrocytes

${ }^{\mathrm{c}}$ Bromelain-treated erythrocytes

${ }^{\mathrm{d}}$ Subtilisin-treated erythrocytes

${ }^{\mathrm{e}}$ Native erythrocytes

${ }^{\mathrm{f}}$ Pronase-treated erythrocytes

${ }^{\mathrm{g}}$ Neuraminidase-treated erythrocytes
} 
erythrocytes but is unable to agglutinate rabbit and human erythrocytes (Lima et al. 2005). Chicken erythrocytes possess high proportion of $N$-acetylneuraminic acid (95-100\%) along with traces of $N$-glycolylneuraminic acid (0-5\%) (Eylar et al. 1962). As compared to chicken, horse and calf erythrocytes, the sialic acid content is largest amongst human erythrocyte which is about twice their amount (Eylar et al. 1962).

Lectins from marine red algae (specially from the order Gigartinales) are more sensitive to enzyme (trypsin or pronase)-treated rabbit and sheep erythrocytes as compared to other animal and human erythrocytes (Hori et al. 1988b). Mild enzymatic treatment of erythrocytes can expose hidden sites on/within erythrocytes surface and allow access to certain lectins. Proteases such as bromelain and pronase digest outer structures made up of protein and glycoprotein and in turn expose antigens that are normally hidden (Cunliffe and Cox 1979). However, haemagglutination titre of purified S. maxima lectin decreases with proteolytic enzyme (trypsin and protease)-treated horse erythrocytes (Shiomi et al. 1980). Carnin (purified agglutinin from Carpopeltis flabellata) does not show any agglutination with trypsin-treated sheep and human ABO erythrocytes (Hori et al. 1987). Upon enzymatic treatment of erythrocyte, agglutination titre increases, if lectin receptors become mobile on membrane surface or decreases upon removal of membrane receptors (Shiomi et al. 1980). Thus, upon treatment of erythrocytes with proteolytic enzymes, the susceptibility of agglutination varies.

De novo exposure of underneath crypt antigens occurs upon protease treatment of erythrocytes as it removes glycol coat from its surface. Trypsin treatment of erythrocytes alters the sialic acid content of erythrocyte sialoglycoproteins (Okamura et al. 2007). Solnins, agglutinins from Solieria robusta, strongly agglutinate rabbit erythrocytes with an enhanced sensitivity towards enzyme (trypsin/pronase)-treated rabbit erythrocytes (Hori et al. 1987). Solnin A, isolectin purified from S. robusta, specifically agglutinates trypsin-treated human blood type A erythrocytes (Hori et al. 1988a). A lectin purified from Gracilaria ornata strongly agglutinates trypsin-treated rabbit erythrocytes but is unable to agglutinate enzyme-treated human erythrocytes (Leite et al. 2005). Lectins from Hypnea japonica, C. flabellata and S. robusta strongly agglutinate trypsin-treated rabbit erythrocytes (Hori et al. 1990). Kappaphycus alvarezii (earlier called Eucheuma cottonii) crude lectin strongly agglutinates enzyme (trypsin and papain)-treated sheep and rabbit erythrocytes as compared to native erythrocytes and is unable to agglutinate chicken and human erythrocytes (Le et al. 2009). Proteolytic enzymes such as papain or bromelain hydrolyse proteins such as membrane sialoglycoprotein (glycophorin A) on the erythrocyte surface leading to reduction in cell surface sialic acid (Lambert et al. 1977; Rogers and Topliss 1983). Neuraminidase treatment of erythrocytes leads to reduction in lectin activity of Solieria chordalis extract; thus, lectin receptor might be sialic acid as neuraminidase treatment of erythrocyte surface can lead to removal of surface sialic acid (Rogers and Topliss 1983). Purified recombinant $B$. triquetrum lectin (rBTL) agglutinates only papain and trypsin-treated rabbit erythrocytes indicating that its ligands are not directly accessible at the cell surface (Nascimento et al. 2015). Crude lectin from A. nana exhibits a high haemagglutination titre with trypsin-treated rabbit erythrocytes (Anam et al. 2017a). Further, carbohydrate specificity of these lectins is the underlying cause for their varied agglutination preferences towards different erythrocytes.

\section{Carbohydrate specificity of lectins from red algae}

Depending on the originating algal species, the hemagglutination-inhibition profiles of crude lectins and lectins purified from red algae are diverse with unique carbohydratebinding specificities and tabulated in Tables 3 and 4, respectively. Haemagglutination activity of lectins from Rhodophyceae species is strongly inhibited by various glycoproteins and glycopeptides which may recognise various membrane-associated complex carbohydrates. Thus, based on their binding preferences towards glycoproteins, lectins from red algae can be categorised as complex-type specific (complex $N$-glycan or complex $O$-glycan or both), high-mannose type specific or both complex and high-mannose glycan specific. However, lectins from few red algae also show specificity towards monosaccharides and their derivatives.

\section{Glycoprotein-specific lectins}

Majority of lectins from red algae are not inhibited by simple sugars constituting the carbohydrate moiety of the glycoprotein; however, they particularly recognise complex carbohydrate structure in the glycoprotein (Tables 3 and 4).

\section{Complex carbohydrate specific}

Some lectins exhibit specificity towards sugar chains of $\mathrm{N}$ glycosidic types (Hori et al. 1986a), whereas a few are $O$ glycan specific (Dinh et al. 2009). Carbohydrate specificity of purified lectins from E. duperreyi (Benevides et al. 1998), Ptilota filicina (Sampaio et al. 1998), Pterocladiella capillacea (Oliveira et al. 2002), G. ornata (Leite et al. 2005) and G. cornea (Lima et al. 2005) is complex and is strongly inhibited by porcine stomach mucin (PSM). PSM is an $O$-linked glycoprotein having terminal GalNAc residues along with galactose and fucose as internal residues (Sampaio et al. 1999). Crude protein extract of Gracilaria fisheri (GPE) has specificity towards porcine stomach mucin, fetuin and bovine albumin with a minimum inhibitory concentration (MIC) value of $1.56,3.12$ and $6.25 \mu \mathrm{g} \mathrm{mL}^{-1}$, respectively (Boonsri et al. 2017). L-GVH exhibits specificity 
Table 3 Carbohydrate specificity of crude lectins from red algae

\begin{tabular}{|c|c|c|}
\hline Red algae & Carbohydrate/glycoprotein specificity & Reference(s) \\
\hline Acanthophora spicifera & $\begin{array}{l}\text { Fetuin, asialofetuin, yeast mannan, porcine stomach thyroglobulin, } \\
\text { bovine submaxillary mucin and asialo bovine submaxillary mucin }\end{array}$ & Dinh et al. (2009) \\
\hline Carpopeltis flabellata & Complex and high-mannose $N$-glycans & Hori et al. (1990) \\
\hline C. flabellata & L-rhamnose, fetuin, $\alpha_{1}$-acid glycoprotein and yeast mannan & Hori et al. (1986a) \\
\hline Eucheuma denticulatum & $\begin{array}{l}\text { Transferrin, asialotransferrin, fetuin, asialofetuin, yeast mannan, } \\
\text { porcine stomach thyroglobulin, bovine submaxillary mucin } \\
\text { and asialo bovine submaxillary mucin }\end{array}$ & Dinh et al. (2009) \\
\hline E. nudum & Fetuin and lactoferrin & Chiles and Bird (1989) \\
\hline Gigartina skottsbergii & Fetuin and bovine submaxillary mucin & De Souza et al. (2007) \\
\hline Gracilaria bursa-pastoris & Fetuin, $\alpha_{1}$-acid glycoprotein and ovomucoid & Hori et al. (1986a) \\
\hline G. euchematoides & $\begin{array}{l}N \text {-acetyl-D-glucosamine, } N \text {-acetyl-D-galactosamine, transferrin, } \\
\text { asialotransferrin, fetuin, asialofetuin, porcine stomach thyroglobulin, } \\
\text { bovine submaxillary mucin and asialo bovine submaxillary mucin }\end{array}$ & Dinh et al. (2009) \\
\hline G. fisheri & Porcine stomach mucin, bovine albumin and fetuin & Boonsri et al. (2017) \\
\hline G. salicornia & $\begin{array}{l}\text { Asialotransferrin, fetuin, asialofetuin, porcine stomach thyroglobulin, } \\
\text { bovine submaxillary mucin and asialo bovine submaxillary mucin }\end{array}$ & Dinh et al. (2009) \\
\hline Halymenia agardhii & Fetuin and lactoferrin & Chiles and Bird (1989) \\
\hline Hypnea boergesenii & $\begin{array}{l}\text { Transferrin, asialotransferrin, fetuin, asialofetuin, porcine stomach } \\
\text { thyroglobulin, bovine submaxillary mucin and asialo bovine } \\
\text { submaxillary mucin }\end{array}$ & Dinh et al. (2009) \\
\hline H. japonica & Fetuin, $\alpha_{1}$-acid glycoprotein and ovomucoid & Hori et al. (1986a) \\
\hline H. nidulans & $\begin{array}{l}\text { Transferrin, asialotransferrin, fetuin, asialofetuin, porcine stomach } \\
\text { thyroglobulin, bovine submaxillary mucin and asialo bovine } \\
\text { submaxillary mucin }\end{array}$ & Dinh et al. (2009) \\
\hline H. valentiae & $\begin{array}{l}\text { Asialotransferrin, fetuin, asialofetuin, porcine stomach thyroglobulin, } \\
\text { bovine submaxillary mucin and asialo bovine submaxillary mucin }\end{array}$ & Dinh et al. (2009) \\
\hline Kappaphycus alvarezii & $\begin{array}{l}\text { Transferrin, asialotransferrin, fetuin, asialofetuin, yeast mannan, } \\
\text { porcine stomach thyroglobulin, bovine submaxillary mucin and } \\
\text { asialo bovine submaxillary mucin }\end{array}$ & Dinh et al. (2009) \\
\hline K. alvarezii & $\begin{array}{l}\text { Fetuin, porcine thyroglobulin, asialo-porcine thyroglobulin, bovine } \\
\text { thyroglobulin, asialo-bovine thyroglobulin, yeast mannan, bovine } \\
\text { submaxillary mucin and asialo-bovine submaxillary mucin }\end{array}$ & Le et al. (2009) \\
\hline K. alvarezii & High-mannose type $N$-glycans & Sato et al. (2011) \\
\hline
\end{tabular}

towards complex-type glycoproteins (asialofetuin, fetuin and thyroglobulin), with highest affinity towards desialylated oligosaccharide chain of asialofetuin as revealed by inhibition assays (Kakita et al. 1999). Asialofetuin and fetuin consist of triantennary $N$-linked glycans possessing terminal galactose and sialic acid, respectively (Sampaio et al. 1999). Ovomucoid consists of highly complex $N$-linked glycans, ovalbumin possesses heterogenous $N$-linked glycans and thyroglobulin exhibits both high-mannose and complex-type $N$ glycans (Sampaio et al. 1999). Purified lectin from Hypnea cervicornis is inhibited only by glycoprotein PSM at an MIC value of $19 \mu \mathrm{g} \mathrm{mL}^{-1}$ (Nascimento et al. 2006). Tichocarpus crinitus (Molchanova et al. 2010) and Georgiella confluens (Souza et al. 2010) purified lectins exhibit complex carbohydrate-binding specificity as its activity is inhibited by glycoprotein fetuin and PSM only. The hemagglutinating activity of the A. callophyllidicola purified lectin is inhibited by complex glycoproteins, fetuin and asialofetuin with an MIC value of 19 and $62 \mu \mathrm{g} \mathrm{mL}^{-1}$, respectively (Shim et al. 2012).
Hypnea japonica crude lectin exhibits specificity only towards complex $N$-glycans, as revealed by haemagglutinationinhibition tests (Hori et al. 1986a). Similar to hypnin A, isohaemagglutinins (hypnin A-1 and A-2) from $H$. japonica do not recognise high-mannose $N$-glycans but exhibit specificity towards some glycoproteins bearing complex-type $\mathrm{N}$ glycans (transferrin, fetuin and $\alpha_{1}$-acid glycoprotein) or $O$ glycans (fetuin and mucin), their desialylated forms and glycopeptides prepared from asialofetuin (Hori et al. 2000). Isolectin (hypnin A3) purified from $H$. japonica exhibits unique and strict specificity towards core ( $\alpha 1-6)$ fucosylated $N$-glycans as revealed by SPR analysis and hapten inhibition assay (Okuyama et al. 2009). Crude lectins from Hypnea valentiae, $H$. boergesenii, $H$. nidulans and Gracilaria salicornia are specific for $O$-glycans as their lectin activity was inhibited by asialofetuin bearing both complex $N$-glycans and $O$-glycans as well as by bovine submaxillary mucin (BSM) and its asialo-derivative bearing $O$-glycans (Dinh et al. 2009). BSM consists of $N$-acetyl neuraminic acid as 
Table 4 Carbohydrate specificity of purified lectins from red algae

\begin{tabular}{l} 
Red algae \\
\hline Aglaothamnion callophyllidicola \\
Bryothamnion seaforthii \\
B. triquetrum \\
B. triquetrum \\
Carpopeltis flabellata \\
Enantiocladia duperreyi
\end{tabular}

Eucheuma amakusaensis

E. cottonii

E. serra

Georgiella confluens

Gracilaria cornea

G. ornata

G. tikvahiae

G. verrucosa

Hypnea cervicornis

H. cervicornis

H. japonica

H. japonica

H. japonica

H. musciformis

Kappaphycus striatum

Palmaria palmata

Pterocladiella capillacea
Carbohydrate/glycoprotein specificity

Reference(s)

Fetuin and asialofetuin

Fetuin, mucin and avidin

Fetuin, mucin and avidin

$N$-glycans and core $\alpha 1$,6-fucosylated octasaccharide

Transferrin, fetuin, $\alpha_{1}$-acid glycoprotein, asialofetuin and yeast mannan

$N$-acetyl-D-galactosamine, D-galactosamine, $o$-nitrophenyl- $\beta$-D-galactopyranoside, lactulose, lactose, $\beta$-lactose, D-melibiose, $p$-nitrophenyl- $\alpha$-D-galactopiranoside, $p$-nitrophenyl- $\beta$-D-galactopiranoside, D-fucose, methyl- $\alpha$-D-galactopiranoside, D-galactose, D-raffinose, methyl- $\beta$-D-galactopiranoside and porcine stomach mucin

$\alpha 1$-Acid glycoprotein, BSA, mucin, $\gamma$-globulin (bovine), fetuin, asialofetuin, ovalbumin, IgM (mouse), thyrogloblin and yeast mannan

$\alpha 1$-Acid glycoprotein, BSA, mucin, $\gamma$-globulin (bovine), fetuin, asialofetuin, ovalbumin, IgM (mouse), thyrogloblin and yeast mannan

High-mannose type (HM) $\mathrm{N}$-glycans

Fetuin and porcine stomach mucin

Fetuin and porcine stomach mucin

Asialofetuin, lactotransferrin, porcine stomach mucin, bovine thyroglobulin and porcine thyroglobulin

$N$-acetylneuraminic acid, $\alpha$-acid glycoprotein, asialofetuin, fetuin, lactoferrin, ovomucoid, asialo bovine submaxillary mucin, bovine submaxillary mucin, human transferring, fetuin glycopeptide A, glycopeptide B and lactoferrin glycopeptide I

Asialofetuin, fetuin and thyroglobulin

D-glucose, D-mannose, D-galactose, methyl- $\alpha$-D-galactopyranoside, L-fucose, $N$-acetyl D-galactosamine, $N$-acetyl D-glucosamine, lactulose, carrageenan, fucoidan, human serotransferrin, desialylated human serotransferrin, $\alpha 1$ acid glycoprotein, desialylated $\alpha 1$ acid glycoprotein, human lactotransferrin, desialylated human lactotransferrin, hen ovomucoid, hen ovalbumin, porcine thyroglobulin, desialylated porcine thyroglobulin, bovine lactotransferrin, desialylated bovine lactotransferrin, bovine fetuine, bovine asialofetuin, bovine submaxillary mucin, porcine stomach mucin, ovine submaxillary mucin and desialylated ovine submaxillary mucin

Porcine stomach mucin

Fetuin, $\alpha_{1}$-acid glycoprotein and ovomucoid

Glycoproteins bearing complex-type $N$-glycans (transferrin, fetuin and K1-acid glycoprotein) or $O$-glycans (fetuin and mucin), their desialylated forms and the glycopeptide prepared from asialofetuin

Core $\alpha 1-6$ fucosylated $N$-glycans and glycoprotein containing core ( $\alpha 1-6)$ fucose

D-glucose, D-mannose, D-galactose, methyl-a-D-galactopyranoside, L-fucose, $N$-acetyl D-galactosamine, $N$-acetyl D-glucosamine, lactulose, carrageenan, fucoidan, human serotransferrin, desialylated human serotransferrin, $\alpha 1$ acid glycoprotein, desialylated $\alpha 1$ acid glycoprotein, human lactotransferrin, desialylated human lactotransferrin, hen ovomucoid, hen ovalbumin, porcine thyroglobulin, desialylated porcine thyroglobulin, bovine lactotransferrin, desialylated bovine lactotransferrin, bovine fetuine, bovine asialofetuin, bovine submaxillary mucin, porcine stomach mucin, ovine submaxillary mucin and desialylated ovine submaxillary mucin

Fetuin, yeast mannan, porcine thyroglobulin, asialo-porcine thyroglobulin, bovine thyroglobulin, asialo-bovine thyroglobulin, bovine submaxillary mucin and asialo-bovine submaxillary mucin

$\mathrm{N}$-acetylneuraminic acid and D-glucuronic acid

Avidin and porcine stomach mucin
Hung et al. (2011)

Shim et al. (2012)

Ainouz et al. (1995)

Ainouz et al. (1995)

Nascimento et al. (2015)

Hori et al. (1987)

Benevides et al. (1998)

Kawakubo et al. (1997), Kawakubo et al. (1999)

Kawakubo et al. (1999)

Hori et al. (2007)

De Souza et al. (2007),

Souza et al. (2010)

Lima et al. (2005)

Leite et al. (2005)

Chiles and Bird (1990)

Kakita et al. (1997)

Nagano et al. (2005)

Nascimento et al. (2006)

Hori et al. (1986a)

Hori et al. (1996b, 2000)

Okuyama et al. (2009)

Nagano et al. (2005)

Kamiya et al. (1982)

Oliveira et al. (2002) 
Table 4 (continued)

\begin{tabular}{|c|c|c|}
\hline Red algae & Carbohydrate/glycoprotein specificity & Reference(s) \\
\hline P. capillacea & Mucin & Silva et al. (2010) \\
\hline Ptilota filicina & $\begin{array}{l}p \text {-Nitrophenyl- } N \text {-acetyl- } \alpha \text {-D-galactoside, } p \text {-nitrophenyl- } N \text {-acetyl- } \beta \text {-D-galactoside, } \\
o \text {-nitrophenyl- } N \text {-acetyl- } \alpha \text {-D-galactoside, } o \text {-nitrophenyl- } \beta \text {-D-fucoside, } \\
p \text {-nitrophenyl- } \alpha \text {-D-galactoside, } p \text {-nitrophenyl- } \beta \text {-D-fucoside, } \\
p \text {-nitrophenyl- } \beta \text {-D-galactoside, } o \text {-nitrophenyl- } N \text {-acetyl- } \beta \text {-D-galactoside, } \\
o \text {-nitrophenyl- } \alpha \text {-D-galactoside, } o \text {-nitrophenyl- } \beta \text {-D-galactoside, lactose, } \\
N \text {-acetyl-galactosamine, melibiose, D-galactose, methyl- } \alpha \text {-D-galactoside, } \\
\text { galactosamine-HCl, methyl- } \beta \text {-D-galactoside, raffinose, } 2 \text {-deoxy-D-galactose, } \\
\text { D-fucose, lactulose, fucoidan, porcine stomach mucin, asialo bovine mucin } \\
\text { and bovine submaxillary gland mucin }\end{array}$ & Sampaio et al. (1998) \\
\hline P. plumosa & $\begin{array}{l}p \text {-nitrophenyl- } \alpha \text {-D-galactoside, } p \text {-nitrophenyl- } \alpha \text {-D-glucoside, } \\
p \text {-nitrophenyl- } \beta \text {-D-fucoside, D-glucose, D-fucose, methyl- } \alpha \text {-D-galactoside, } \\
p \text {-nitropheyl- } \beta \text {-D-galactoside, } p \text {-nitrophenyl- } \beta \text {-D-glucoside, D-galactose, } \\
\text { L-fucose, } o \text {-nitrophenyl- } \alpha \text {-D-galactoside, } o \text {-nitrophenyl- } \beta \text {-D-fucoside, } \\
\text { 2-deoxy-D-glucose, methyl- } \beta \text {-D-galactoside, D-arabinose, melibiose, } \\
\text { raffinose, } \alpha \text {-lactose, } o \text {-nitrophenyl- } \beta \text {-D-galactoside, lactulose, } \\
\text { glucosamine- } \mathrm{HCl} \text {, rhamnose and } N \text {-acetyl-glucosamine }\end{array}$ & Sampaio et al. (2002) \\
\hline P. serrata & $\begin{array}{l}o \text {-Nitrophenyl- } N \text {-acetyl- } \alpha \text {-D-galactoside, } p \text {-nitrophenyl- } N \text {-acetyl- } \beta \text {-D-galactoside, } \\
\text { lactose, } o \text {-nitrophenyl- } N \text {-acetyl- } \beta \text {-D-galactoside, } \\
p \text {-nitrophenyl- } N \text {-acetyl- } \alpha \text {-D-galactoside, } p \text {-nitrophenyl- } \beta \text {-D-fucoside, } \\
o \text {-nitrophenyl- } \beta \text {-D-fucoside, } N \text {-acetyl-galactosamine, methyl- } \alpha \text {-D-galactoside, } \\
\text { methyl- } \beta \text {-D-galactoside, D-galactose, melibiose, D-fucose, } \\
o \text {-nitrophenyl- } \alpha \text {-D-galactoside, } p \text {-nitrophenyl- } \beta \text {-D-galactoside, } \\
\text { galactosamine-HCl, raffinose, lactulose, } o \text {-nitrophenyl- } \beta \text {-D-galactoside, } \\
p \text {-nitrophenyl- } \alpha \text {-D-galactoside, } 2 \text {-deoxy-D-galactose, fucoidan, porcine } \\
\text { stomach mucin, asialo bovine mucin, asialofetuin and bovine submaxillary } \\
\text { gland mucin }\end{array}$ & Sampaio et al. (1999) \\
\hline Solieria chordalis & $\begin{array}{l}\text { Fetuin, bovine submaxillary gland mucin, porcine mucin, } \alpha \text {-D(+)-melibiose } \\
\text { and human MN sialoglycoprotein }\end{array}$ & Rogers and Toplis (1983) \\
\hline S. filiformis & Mannose oligosaccharides & Chaves et al. (2017) \\
\hline S. robusta & $\begin{array}{l}\text { Fetuin, asialotransferrin, asialofetuin, asialo- } \alpha \text {-acid glycoprotein, yeast mannan, } \\
\text { ovalbumin, glycopeptide-fraction from fetuin and } N \text {-glycopeptide from yeast } \\
\text { mannan }\end{array}$ & Hori et al. (1988a) \\
\hline S. robusta & Complex and high-mannose $N$-glycans & Hori et al. (1996b) \\
\hline Tichocarpus crinitus & Porcine stomach mucin and fetuin & Molchanova et al. (2010) \\
\hline Vidalia obtusiloba & $\begin{array}{l}N \text {-acetyl-galactosamine, D-galactosamine, D-galactose, } \alpha \text {-lactose and porcine } \\
\text { stomach mucin }\end{array}$ & Melo et al. (2004) \\
\hline
\end{tabular}

terminal residue linked to GalNAc (Sampaio et al. 1999). As fetuins are carrier proteins in bloodstream (Bies et al. 2004) and asialofetuin-labelled liposomes are useful for receptormediated transfer of DNA sequence to mouse liver cells (Hara et al. 1995), thus fetuin/asialofetuin-specific lectins could be useful in drug delivery studies. A rare specificity is exhibited by $S$. chordalis purified agglutinin towards receptor incorporated in $\mathrm{O}(2 \rightarrow 6)$ glucoside of sialic acid and 2acetamido-2-deoxy-D-galactopyranose as revealed by haemagglutination inhibition by sialoglycoproteins fetuin, BSM and PSM (Rogers and Topliss 1983). Preference of S. chordalis purified lectin towards subterminal GalNAc instead of Gal residue suggests the role of $N$-acetyl group in conferring rigid conformation on penultimate sugar, thus leading to stabilizing influence on terminal residue linkage (Rogers and Topliss 1983).

\section{High-mannose specific}

The genus Eucheuma is a valuable source of lectin proteins as its several species possess high yields of lectins which are homologous between species (Kawakubo et al. 1999). Eucheuma serra purified agglutinin, ESA-2, is the only lectin that has strict oligosaccharide binding specificity towards high-mannose $N$-glycans along with terminal GlcNAc residues without binding to monosaccharides and core pentasaccharide (Hori et al. 2007). Carbohydrate inhibition studies revealed that $K$. alvarezii (earlier called $E$. cottonii) and Eucheuma denticulatum crude lectins are specific for highmannose $N$-glycans as yeast mannan bearing high-mannose $N$-glycans was most inhibitory along with porcine stomach thyroglobulin (PTG) bearing both high-mannose and complex $N$-glycans was also inhibitory (Dinh et al. 2009). 
Kappaphycus alvarezii crude agglutinin (KAA) preferentially recognises high-mannose type $N$-linked glycoproteins (Le et al. 2009). Consistent with native KAA, recombinant KAA (His-rKAA-1, rKAA-1) lectins from Kappaphycus alverezii also shows specificity towards high-mannose glycoproteins such as yeast mannan, PTG, asialo-PTG, BSM, asialo-BSM, fetuin, asialofetuin, transferrin and asialo-transferrin (Hirayama et al. 2016).

Purified isolectins from Kappaphycus striatum, KSA-1, KSA-2 and KSA-3 (Hung et al. 2011), and K. alvarezii, KAA-1 and KAA-2 (Le et al. 2009), have special affinity towards high-mannose $\mathrm{N}$-glycans as revealed by haemagglutination inhibition analysis. High-mannose type $\mathrm{N}$-glycan possessing molecules such as yeast mannan, porcine and bovine thyroglobulin and their asialo-derivatives are highly inhibitory towards haemagglutination activity of Kappaphycus isolectins (Le et al. 2009; Hung et al. 2011). Moderate inhibition of Kappaphycus isolectins by $O$ linked glycan glycoproteins (fetuin and BSM) is due to non-specific interaction between lectin and $O$-linked glycoproteins (Le et al. 2009; Hung et al. 2011). KSA-2 isolectin specifically interacts with extended carbohydrate structure with a minimal length of tetrasaccharide, $\operatorname{Man}(\alpha 1-3) \operatorname{Man}(\alpha 1-6) \operatorname{Man}(\beta 1-4)$ GlcNAc (Hung et al. 2011). GRFT purified from Griffithsia sp. exhibits specificity towards $\alpha(1,2)$ mannobiose (Moulaei et al. 2010, 2015). The hemagglutination activitiy of isolectins from E. denticulatum (EDA-1, EDA-2 and EDA-3) is commonly inhibited by glycoproteins bearing high-mannose $\mathrm{N}$-glycans but not by monosaccharides such as mannose (Hung et al. 2015). In a direct binding experiment with pyridylaminated oligosaccharides, an isolectin EDA-2 exclusively binds to high-mannose type $N$-glycans but not to other glycans that include complex types and a core pentasaccharide of $\mathrm{N}$-glycans, indicating that it recognises the branched oligomannoside moiety (Hung et al. 2015). Molecular docking calculations and favourable binding energies reveal that purified isolectins (SfL-1 and SfL-2) from $S$. filiformis specifically bind to $3 \alpha, 6 \alpha$ mannopentose oligosaccharides, thus exhibits specificity towards highmannose oligosaccharides (Chaves et al. 2017).

\section{Both high-mannose and complex glycoprotein specific}

Carpopeltis flabellata (Hori et al. 1986a, 1987) and S. robusta (Hori et al. 1988a) purified lectins recognise both highmannose $N$-glycans and complex $N$-glycans. Purified agglutinins from marine red algae E. serra (ESA-1 and ESA-2), E. amakusaensis (EAA-1, EAA-2 and EAA-3) and E. cottonii (ECA-1 and ECA-2) inhibit complex specific glycans but have preferential affinity for glycoproteins bearing high-mannose type $N$-glycans such as thyrogloblin and yeast mannan (Kawakubo et al., 1997, 1999).

\section{Simple sugar-specific lectins}

Mostly red algae agglutinins have affinity towards various glycoconjugates, whereas lectin-induced agglutination is usually unaffected with monosaccharides as inhibitors. However, a few lectins from red algae act as an exception by exhibiting specificity towards varied monosaccharides. Crude haemagglutinin from C. flabellata is inhibited by Lrhamnose (Hori et al. 1986a). Purified lectins from marine algae P. serrata, PSL (Sampaio et al. 1999), P. filicina, PFL (Sampaio et al. 1998) and V. obtusiloba (Melo et al. 2004) are strongly inhibited by simple sugar (galactose) and their derivatives and by glycoprotein PSM. A welldefined monosaccharide binding site is possessed by PFL and PSL which recognise specific arrangement in more complex glycans where galactose occurs (Sampaio et al., 1998, 1999). Enantiocladia duperreyi purified lectininduced agglutination is inhibited by simple sugars (glucose and fucose) and their derivatives (Benevides et al. 1998). Enantiocladia duperreyi lectin exhibits preference for bromelain-treated human blood group $\mathrm{O}$ erythrocytes which has D-fucose as its sugar determinant (Benevides et al. 1998). Enantiocladia duperreyi lectin thus has $\mathrm{H}$ antigen preference as also indicated by inhibitory effect of PSM, which is a fucose-carrying glycoprotein (Benevides et al. 1998). Inhibition of purified lectins from E. duperreyi (Benevides et al. 1998), P. filicina (Sampaio et al. 1998) and P. serrata (Sampaio et al. 1999) by nitophenyl-galactosides is stronger than methyl-D-galactosides; thus, these lectins possess hydrophobic region in vicinity of its carbohydrate-binding site (Mo and Goldstein 1994). Purified lectin from G. tikvahiae also exhibits affinity towards $N$-acetylneuraminic acid along with its glycoconjugate, $N$-acetylneuraminic acid- $(2 \rightarrow$ 3)-lactose (Chiles and Bird 1990). Purified lectins from few algal species such as $P$. serrata (Sampaio et al. 1999) and P. filicina (Sampaio et al. 1998) react with more extended structures/polysaccharides (such as fucoidan) than monosaccharides (L-fucose). Purified rBTL is the first lectin that exhibits strict specificity towards core 6-fucose; however, it shows no binding to $\mathrm{ABO}$, Lewis epitopes or to 3 -fucosylated $\mathrm{N}$-glycan core (Nascimento et al. 2015).

\section{Characteristics of lectins from red algae}

Marine macroalgae lectins have been widely isolated and characterised particularly the members of Rhodophyceae (Hori et al. 1990). A panorama of lectins from red algae, purified and characterised over decades, has been tabulated in Table 5. 


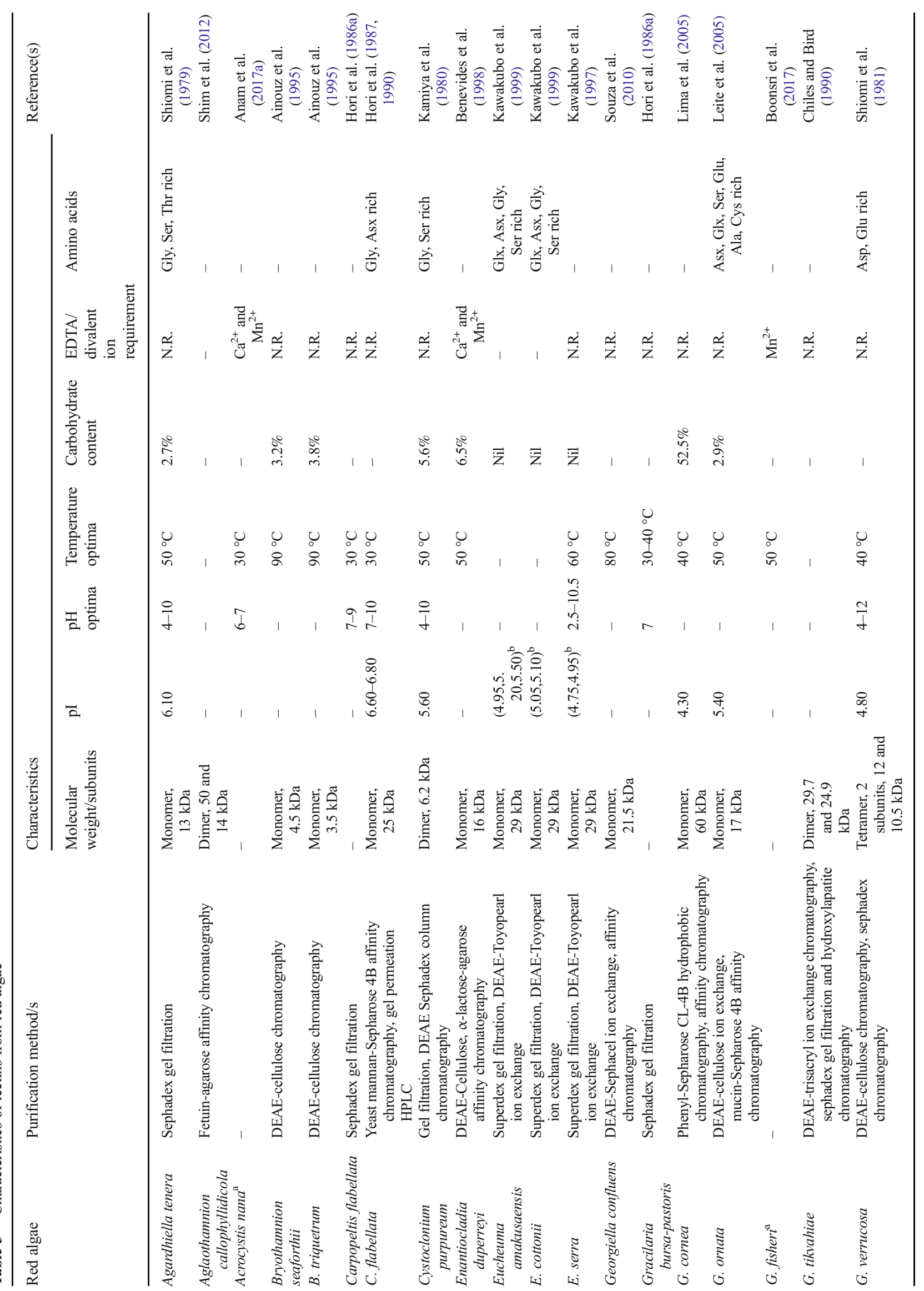




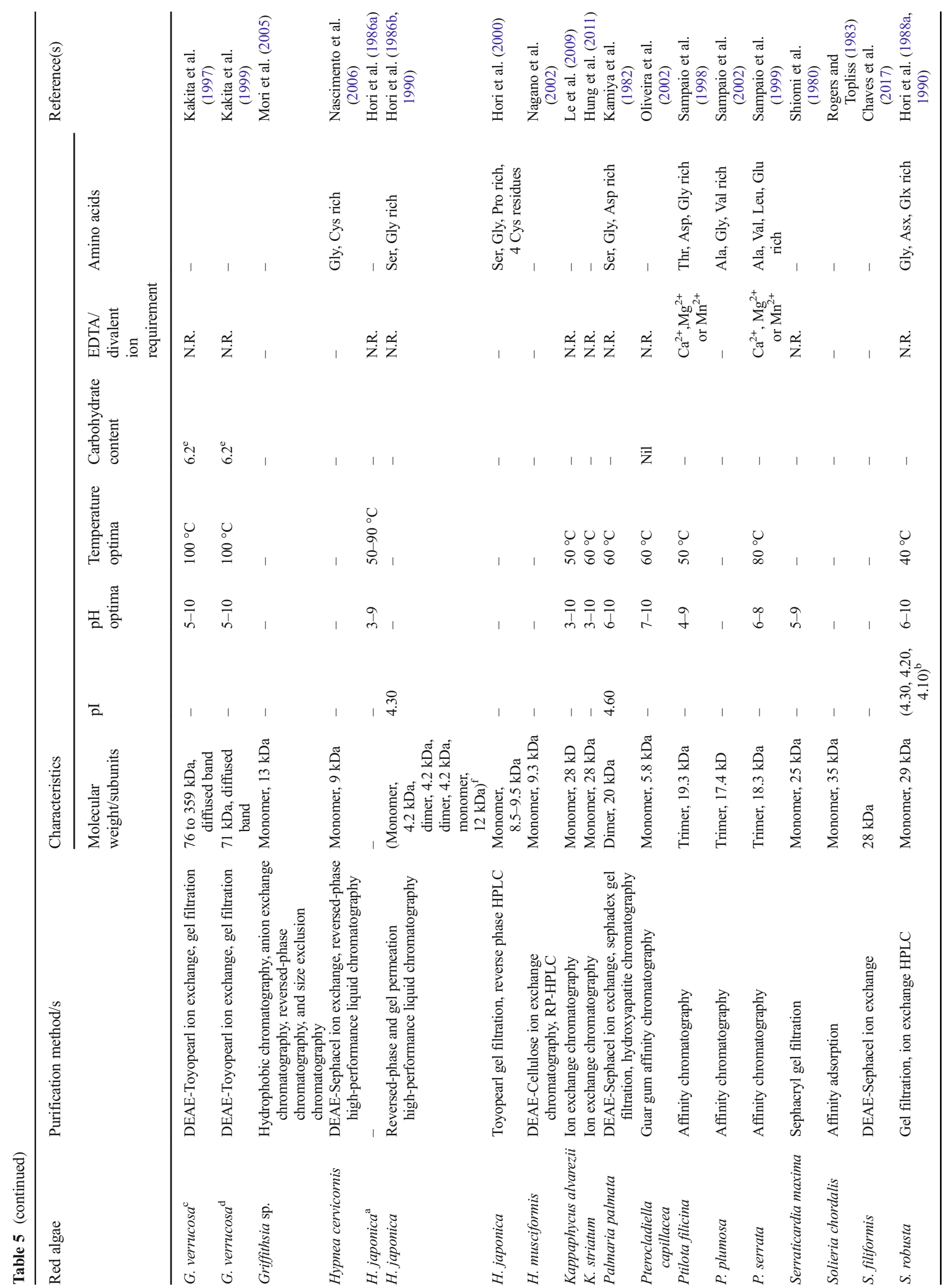




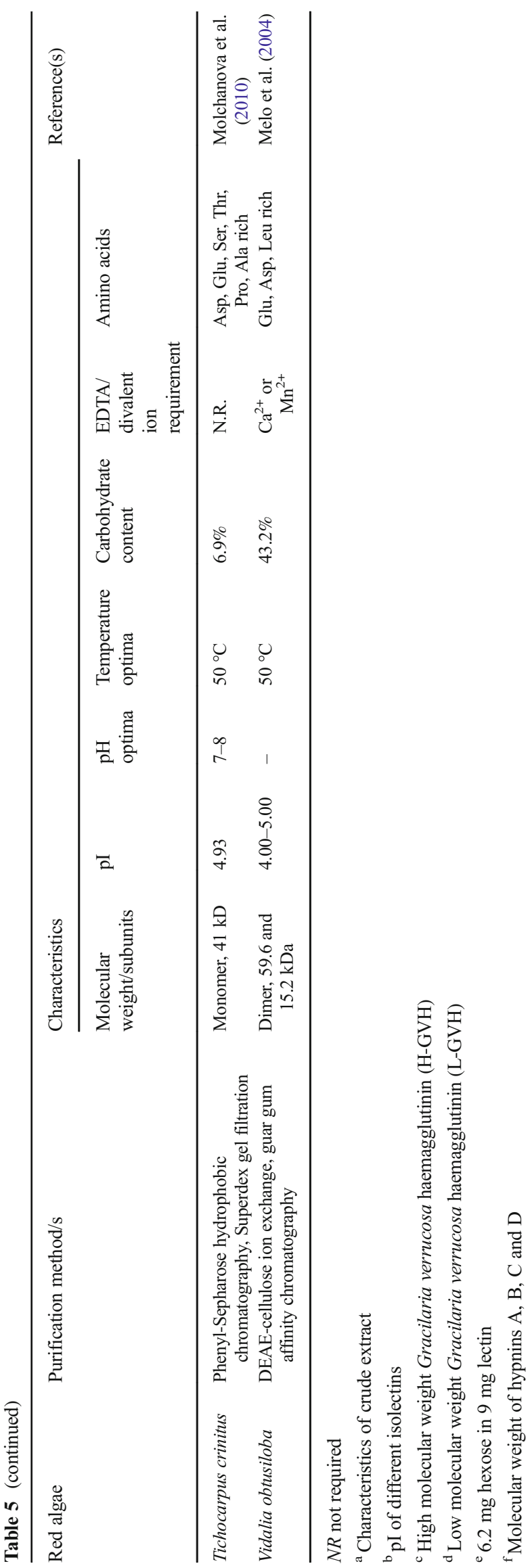

\section{Purification methods}

Lectins from red algae can be purified by either multistep chromatographic techniques or single-step affinity chromatography (Table 5). Owing to carbohydrate specificity of lectins, they are mostly purified by single-step affinity chromatography. Lectins from P. filicina (Sampaio et al. 1998) and P. serrata (Sampaio et al. 1999) have been isolated by affinity chromatography onto a cross-linked guar gum and 95-fold and 1.8-fold purification, respectively, was achieved. Pterocladiella capillacea lectin has been purified by affinity chromatography onto a guar gum column with a purification fold of 14.5 and recovery yield of $27.4 \%$ (Oliveira et al. 2002). Ptilota plumosa lectin (PPL) has been purified from algal extracts by affinity chromatography using Sephadex G-200 column and a purification fold of 212 and high specific activity of 46,282 was obtained (Sampaio et al. 2002). Fetuinspecific lectin from A. callophyllidicola has been purified by agarose-bound fetuin affinity chromatography (Shim et al. 2012).

A lectin has been purified from the seaweed Gracilaria cornea by combination of phenyl-Sepharose CL-4B hydrophobic interaction chromatography followed by affinity chromatography using immobilised mucin (Lima et al. 2005). Lectin from red marine algae $V$. obtusiloba has been purified by combination of ion exchange chromatography on DEAEcellulose and affinity chromatography onto a cross-linked guar gum column (Melo et al. 2004). Gracilaria ornata lectin has been purified by DEAE-cellulose ion exchange chromatography and mucin-Sepharose 4B affinity chromatography (Leite et al. 2005). A lectin from Antarctic seaweed G. confluens has been purified by ion exchange followed by affinity chromatography using immobilised PSM (Souza et al. 2010). Tichocarpus crinitus lectin has been purified by phenyl-Sepharose hydrophobic chromatography and gel exclusion chromatography (Molchanova et al. 2010). Recently, isolectins from $S$. filiformis have been purified by DEAESephacel ion exchange chromatography (Chaves et al. 2017).

\section{Isoelectric point and amino acid content}

Lectins from red algae have low isoelectric points (pIs), usually in the range of 4-6 (Table 5). Red algal lectins usually have high contents of acidic (aspartic acid and glutamic acid) and hydroxyl (serine and threonine) amino acids and low levels of basic amino acids such as arginine, histidine and lysine (Hori et al. 1990; Okamoto et al. 1990; Sampaio et al. 1999). The large proportion of acidic amino acids accounts for the acidic $\mathrm{pI}$ in lectins from some red algae species such as S. filiformis (Benevides et al. 1996) and V. obtusiloba (Melo et al. 2004). Vidalia obtusiloba lectin is rich in acidic amino acids (glutamic and aspartic acids) and in the hydrophobic amino acid (leucine) but has a low content of cystine and 
methionine (Melo et al. 2004). Tichocarpus crinitus lectin has high proline content along with acidic and hydroxyl amino acids (Molchanova et al. 2010).

Amino acid composition of $H$. cervicornis lectin (Nascimento et al. 2006) revealed high content of cysteine (15.5 mol\%) and low serine $(4.4 \mathrm{~mol} \%)$, similar to the H. musciformis lectin (15.2 Cys, 5.4 Ser residues per mol). However, H. japonica isolectins contained lower Cys (4.4 mol\%) and higher Ser (15.5 mol\%) (Hori et al. 2000). Similarly, B. triquetrum lectin contained lower Cys (3.6 mol\%) and higher Ser (16.3 mol\%) (Calvete et al. 2000). It seems that amino acid content varies in red algae lectins. Further, H. cervicornis lectin is involved in formation of seven intramolecular disulphide bonds as lectin consists of 14 cysteines per molecule (Nascimento et al. 2006). Amino acid sequence of hypnins revealed that hypnin A-1 and A-2 polypeptides are composed of 90 amino acid residues including four half cysteines which is involved in formation of two intrachain disulphide bonds (Hori et al. 2000). Reduction and Spyridylethylation of haemagglutinin (hypnin A-1 and A-2) result in loss of activity, which indicates the involvement of disulphide bonds in maintaining haemagglutination activity of each haemagglutinin (Hori et al. 2000). The sequence of both the agglutinins varies at three positions, with Leu19, Ser31 and Tyr52 of hypnin A-2 as compared to Pro19, Arg31 and Phe52 of hypnin A-1 (Hori et al. 2000). Gracilaria ornata lectin also consists of high amount of cysteine, $7.79 \mathrm{~mol} \%$ (Leite et al. 2005). However, disulphide bonds might not be involved in subunit association of Cystoclonium purpureum (Kamiya et al. 1980), G. verrucosa (Shiomi et al. 1981) and Palmaria palmata (Kamiya et al. 1982) agglutinins as indicated by the absence of half cystine or cysteine in their amino acid content.

\section{Molecular weight}

Lectins from red algae are usually low molecular weight proteins and exist in monomeric form such as lectins from C. flabellata, S. robusta (Hori et al. 1990), B. seaforthii, B. triquetrum (Ainouz et al. 1995), P. capillacea (Oliveira et al. 2002), H. musciformis (Nagano et al. 2002), G. ornata (Leite et al. 2005), G. cornea (Lima et al. 2005), Griffithsia sp. (Mori et al. 2005; Moulaei et al. 2010, 2015), K. alvarezii (Le et al. 2009), T. crinitus (Molchanova et al. 2010), G. confluens (Souza et al. 2010) and K. striatum (Hung et al. 2011). However, some marine red algal lectins have dimeric structures, such as P. palmata (Kamiya et al. 1982) and V. obtusiloba (Melo et al. 2004); trimeric, such as P. filicina (Sampaio et al. 1998), P. serrata (Sampaio et al. 1999), Ptilota plumosa and Ptilota gunneri (Sampaio et al. 2002); or tetrameric structures, such as G. verrucosa (Shiomi et al. 1981). Lectin from A. callophyllidicola is dimeric with subunits having molecular weights of 50 and $14 \mathrm{kDa}$ (Shim et al. 2012). Isolectins, SfL-1 and SfL-2, from $S$. filiformis have a molecular mass of 27,552 and 27,985 Da, respectively (Chaves et al. 2017).

On the basis of their biochemical properties, Rogers and Hori (1993) categorised red algae lectins into three types: (a) glycoprotein-specific low molecular weight proteins with no requirement of divalent cations, (b) monosaccharide and related small molecule-specific intermediate molecular weight proteins with no requirement of divalent cations and (c) monosaccharide-specific large lectins (MW $>64,000$ ) with requirement of divalent cations. Amongst all red algae lectins reported till now, agglutinins from $B$. seaforthii and $B$. triquetrum have lowest molecular masses 3500 and $4500 \mathrm{Da}$, respectively (Ainouz et al. 1995). A 41-kDa lectin (GVA-1) has been reported from G. verrucosa, which is a key species in Japan as food source or raw material of agar-agar (Shiomi et al. 1981). H-GVH has large molecular size $(480 \mathrm{kD})$ as revealed by gel filtration analysis and is not a classical lectin but a sulfated polysaccharide hemagglutinin and the sulfated polysaccharide moiety of it plays a role in hemagglutination activity (Kakita et al. 1997).

\section{pH and temperature}

Haemagglutination activity of lectins from marine red algae such as K. alvarezii (Le et al. 2009), A. tenera (Shiomi et al. 1979), C. purpureum (Kamiya et al. 1980) and G. verrucosa (Shiomi et al. 1981) lectins is stable over a broad $\mathrm{pH}$ range. Eucheuma serra isolectins also exhibit lectin activity over broad pH range of 2.5-10.5 (Kawakubo et al. 1997). Lectins from few red algae species such as P. serrata (Sampaio et al. 1999), Gracilaria bursa-pastoris (Hori et al. 1986a), T. crinitus (Molchanova et al. 2010) and A. nana (Anam et al. 2017a) exhibit activity near neutral $\mathrm{pH}$ range (6-8) with loss of activity beyond/above this range.

Haemagglutination activity of lectins from $P$. capillacea (Oliveira et al. 2002), G. ornata (Leite et al. 2005), $K$. alvarezii (Le et al. 2009) and crude protein extract from G. fisheri (Boonsri et al. 2017) is stable till $50^{\circ} \mathrm{C}$ temperature. Lectins from C. flabellata (Hori et al. 1986a, 1990) and A. nana (Anam et al. 2017a) are stable only up to $30{ }^{\circ} \mathrm{C}$ temperature. Haemagglutinins from several red algae species such as A. tenera, C. purpureum, C. flabellata and $V$. obtusiloba are thermosensitive with total or almost total activity loss when heated to temperatures equal to or above $60{ }^{\circ} \mathrm{C}$ (Shiomi et al. 1979; Kamiya et al. 1980; Hori et al. 1987; Melo et al. 2004). Pterocladiella capillacea lectin activity is unstable at high temperature, as its activity is lost at $60{ }^{\circ} \mathrm{C}$ (Fabregas et al. 1992). However, a few red algae lectins are thermostable or thermoresistant, such as haemagglutinins from B. seaforthii, B. triquetrum (Ainouz et al. 1995), G. verrucosa (Kakita et al. 1997, 1999), H. japonica (Hori et al. 1986b), P. serrata (Sampaio et al. 1999) and G. confluens (Souza et al. 2010). 


\section{Effect of metal ions}

Red algae lectins usually do not require divalent cations for maintenance of their haemagglutination activity; however, a few lectins isolated from the red algae such as Plumaria elegans, P. serrata (Rogers et al. 1990), P. filicina (Sampaio et al. 1998), E. duperreyi (Benevides et al. 1998), and V. obtusiloba (Melo et al. 2004) are dependent on metals, like $\mathrm{Ca}^{2+}, \mathrm{Mn}^{2+}$ and $\mathrm{Mg}^{2+}$. Crude lectin extract from A. nana requires divalent cations $\mathrm{Ca}^{2+}$ and $\mathrm{Mn}^{2+}$ for its haemagglutination activity (Anam et al. 2017a). Thus, metal requirement is not a general characteristic of red algae lectins; however, a few lectins are metalloproteins.

\section{Carbohydrate content}

A few Rhodophyceae lectins are glycoproteins with varied carbohydrate content; however, some lectins possess no carbohydrate content (Table 5). Lectins from C. purpureum (Kamiya et al. 1980), G. bursa-pastoris (Okamoto et al. 1990), E. duperreyi (Benevides et al. 1998), G. ornata (Leite et al. 2005) and T. crinitus (Molchanova et al. 2010) are glycoproteins. Carbohydrate content of lectins from red algae can be as low as $2.7 \%$ in A. tenera lectin (Shiomi et al. 1979) or $2.9 \%$ in G. ornata lectin (Leite et al. 2005) or as high as $43 \%$ in V. obtusiloba lectin (Melo et al. 2004) or $52.5 \%$ in G. cornea lectin (Lima et al. 2005).

\section{Structure of red algal lectins}

The structural analysis of lectins helps in investigation of their potential applications in various fields. Amongst lectins from red marine algae, the primary structure of $B$. triquetrum lectin (BTL) was the first to be determined (Calvete et al. 2000). Structural characterization of $B$. triquetrum lectin interaction with the octasaccharide depicts an extended epitope for recognition which includes the fucose residue, the distal GlcNAc and one mannose residue as revealed by STD-NMR (Nascimento et al. 2015). Amongst lectins from other red algal species, crystal structure of griffithsin lectin has been thoroughly investigated which is a stable homodimer where each subunit contains 121 amino acids (Ziolkowska et al. 2006, 2007a, 2007b). Crystal structure of carbohydrate free lectin from Griffithsia sp. and its interaction with specific glycan molecules such as mannose, $N$-acetylglucosamine, $1 \rightarrow 6 \alpha$ mannobiose and maltose have been determined through $\mathrm{X}$ ray crystallography, isothermal titration calorimetry and molecular modeling (Ziolkowska et al. 2006; 2007a, b). The domain-swapped dimer of griffithsin molecule involves that first two $\beta$-strands of one chain are connected with ten strands of the other chain and vice versa (Ziolkowska et al. 2006). GRFT-glycan complex analysis revealed that three almost identical carbohydrate-binding sites (separated by approximately $15 \AA$ ) occur on each monomer of GRFT, each interacting with a monosaccharide and thus in turn six principal sites occur in the dimer (Ziolkowska et al. 2006). GRFTglucose (Ziolkowska et al. 2006) and GRFT-mannose (Ziolkowska et al. 2007a) interaction studies revealed the difference in the orientation of the $\mathrm{O} 1$ atom. The interaction heats for glucose (1.5-fold decrease) and $\mathrm{N}$-acetylglucosamine (2fold decrease) are comparable to that of mannose as revealed by isothermal titration calorimetry binding experiments with GRFT (Ziolkowska et al. 2007a, 2007b). However, the saturation of binding does not occur in case of GRFT-glucose and GRFT- $N$-acetylglucosamine (Ziolkowska et al. 2007b), whereas GRFT-mannose binding is fully saturable (Ziolkowska et al. 2007a). GRFT-oligosaccharide interaction studies revealed that it preferentially interacts with terminal sugar and all binding sites have an aspartic acid (Asp) residue which makes extensive contacts with the sugar (Ziolkowska et al. 2007a). Hydrogen bonding interactions occur amongst aspartic acid residue (Asp30, Asp70 and Asp112) and O5 and O6 of mannose (Ziolkowska et al. 2006).

Further, Moulaei et al. (2010) created a monomeric version of GRFT (mGRFT), and mGRFT-Man9 complex studies revealed that two of the three arms of nonamannoside bound to two of the three mannose binding sites in which the terminal mannose units on the D1 and D2 arms are bound to sites 3 and 1 , respectively, and further in a transient interaction, third mannose binding site (site 2) is occupied by the D2 arm of another nonamannoside. However, Ziolkowska et al. (2007a) proposed that all three arms of Man9 $\mathrm{GlcNAc}_{2}$ could interact with GRFT with a one-to-one stoichiometry and thus these interaction studies were inconsistent with Moulaei et al. (2010). Xue et al. (2013) constructed a series of mutants wherein two monomers were connected by an amino acid linker and expressed to form an obligate dimer to understand the importance of having three binding sites on each monomer. They compared a mutant (with three of the binding sites in one of the subunits mutated to remove binding to mannose) and another mutant (with all six mannose binding sites mutated) to a construct having all six binding sites, three from each monomer (Xue et al. 2013). Binding studies revealed that the mutant lacking all mannose binding sites did not bind gp120; however, the obligate dimer and construct containing mannose binding sites on only one subunit bound to gp120 with comparable affinities (Xue et al. 2013). The structural and biochemical studies involving mode of action and construction of lectin analog from Griffithsia sp. further help in laying emphasis over its therapeutic potential (Lusvarghi and Bewley 2016).

The primary structure of lectin from $K$. alvarezii (KAA-2) has been revealed through peptide mapping and complementary DNA (cDNA) cloning which reveals four internal tandem-repeat domains in a molecule (Hirayama et al. 2016). Similar to native KAA lectin, His-tagged rKAA-1 
and rKAA- 1 specifically bind to high-mannose $N$-glycans with an exposed $\alpha 1-3$ mannose in the D2 arm as revealed by oligosaccharide binding analysis by a centrifugal ultrafiltration-HPLC method with 27 pyridylaminated oligosaccharides (Hirayama et al. 2016). Primary structure of two isolectins from S. filiformis (SfL-1 and SfL-2) consisting of four tandem-repeat domains with approximately 67 amino acids has been recently determined (Chaves et al. 2017). Both isolectins exhibit $82 \%$ sequence identity towards each other and are composed of two $\beta$ barrel-like domains formed by five anti-parallel $\beta$-strands, which are connected by a short peptide linker (Chaves et al. 2017). Molecular docking calculations suggest that amino acid residues $\mathrm{W}_{152} \mathrm{G}_{153} \mathrm{G}_{154}, \mathrm{R}_{236}$ and $\mathrm{E}_{264} \mathrm{G}_{265} \mathrm{P}_{266}$ are present in SfL-1 and SfL-2 and are involved in carbohydrate-binding (Chaves et al. 2017).

\section{Biomedical potential of red algal lectins}

Carbohydrate specificity of Rhodophyceae lectins is the underlying basis for their varied activities such as anti-viral, anticancer and anti-inflammatory and thus has potential for diagnosis and selective treatment of these diseases. The percentile potential data of red algae lectins against various targets is shown in Fig. 1 which signifies maximum reports on its anti-HIV activities. The biomedical applications of red algal lectins are given in Table 6 .

\section{Anti-human immunodeficiency virus}

Human immunodeficiency virus (HIV) has exerted an enormous toll on global human health, and with no vaccine currently available, the focus lies on development of alternate effective measures targeting virus entry point and virus-host interaction stages, to halt HIV infections. Griffithsin (GRFT), a lectin derived from Griffithsia sp., has high affinity towards mannose-rich $N$-linked glycans and has strong anti-viral activity against HIV with inhibitory values in the low picomolar range (Mori et al. 2005; Emau et al. 2007; O'Keefe et al. 2009). Grifonin-1 (GRFN-1), an 18-residue peptide derived from $\beta$-sheet core of griffithsin, exhibits an anti-viral activity and low toxicity in vitro, which suggests it a potent topical or systemic agent against HIV (Micevicz et al., 2010). Mechanism of action (MOA) of GRFT involves binding to high-mannose oligosaccharides on gp120 and thus targeting viral entry. GRFT prevents gp120 interaction with HIV coreceptors but does not impede binding of HIV to CD4 (Alexandre et al. 2011). HIV aggregation via multivalent interactions between GRFT and gp120 oligosaccharides occurs owing to dimeric nature of GRFT, with three carbohydratebinding sites per monomer (Moulaei et al. 2010). GRFT inhibits HIV gp120 binding to DC-SIGN and inhibits DCSIGN-mediated HIV transfer to CD4+ T lymphocytes along with removal of gp120 from gp120-DC-SIGN complex (Hoorelbeke et al. 2013). GRFT can act as a systemic antiviral therapeutic agent against enveloped viruses as experimental studies reveals that subcutaneous GRFT administration in rodent species (mouse and guinea pig) accumulates to relevant therapeutic conditions tolerated with minimal toxicity (Barton et al. 2014). Further, in vitro assessment of the toxicological effects of GRFT on isolated mouse peripheral blood mononuclear cells (mPBMCs) and in vivo murine model reveals that GRFT is an outstanding microbicide candidate as no toxicities are reported (Kouokam et al. 2016). Thus, an excellent in vitro and in vivo safety profile for GRFT in the murine model suggests it as a powerful weapon in the fight against human immunodeficiency virus-1 (HIV-1) and other bloodborne enveloped viruses (Kouokam et al. 2016). Thus, GRFT is a potent and broad-spectrum anti-viral agent, which can act as a promising microbicide candidate to prevent HIV acquisition. Recently, Barton et al. (2016) indicated that the pharmacokinetic profile of GRFT depends on the route of

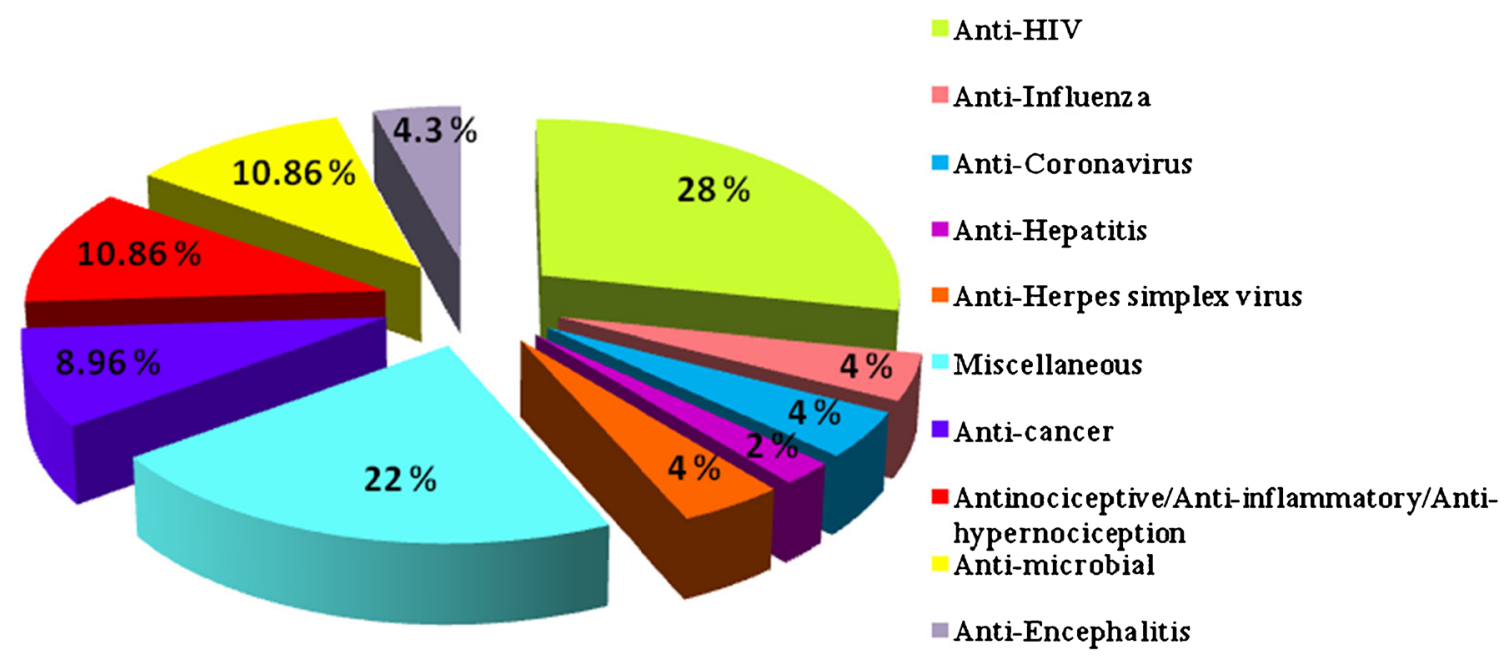

Fig. 1 Percentile data on the biomedical potential of Rhodophyceae lectins against various diseases. Data survey from various internet sources 
Table 6 Biomedical potential of red algae lectins

\begin{tabular}{|c|c|c|}
\hline Red algae & Biomedical application/s & Reference(s) \\
\hline Acrocystis nana & Anti-cancer & Anam et al. (2017b) \\
\hline Amansia multifida & Anti-nociceptive & Neves et al. (2007) \\
\hline \multirow[t]{2}{*}{ Bryothamnion seaforthii } & Anti-cancer & Conrado et al. (2012) \\
\hline & Pro-healing & Nascimento-Neto et al. (2012) \\
\hline B. triquetrum & Anti-cancer & Nascimento et al. (2015) \\
\hline Carpopeltis flabellata & Mitogenic & Hori et al. (1987) \\
\hline \multirow[t]{4}{*}{ Eucheuma serra } & Anti-influenza & Sato et al. (2015) \\
\hline & Anti-cancer & Sugahara et al. (2001), Hayashi et al. (2012) \\
\hline & Anti-bacterial & Liao et al. (2003) \\
\hline & Mitogenic & Kawakubo et al. (1997) \\
\hline Galaxaura marginata & Anti-bacterial & Liao et al. (2003) \\
\hline Gracilaria bursa-pastoris & Mitogenic & Okamoto et al. (1990) \\
\hline G. cornea & Acaricidal & Lima et al. (2005) \\
\hline G. fisheri & Anti-bacterial & Boonsri et al. (2017) \\
\hline G. tikvahiae & Mitogenic & Bird et al. (1993) \\
\hline G. verrucosa & Mitogenic & Bird et al. (1993) \\
\hline \multirow[t]{7}{*}{ Griffithsia sp. } & $\begin{array}{l}\text { Anti-human immunodeficiency } \\
\text { virus }\end{array}$ & $\begin{array}{l}\text { Mori et al. (2005), Emau et al. (2007), O'Keefe et al. (2009), } \\
\text { Micevicz et al. (2010), Moulaei et al. (2010), Alexandre et al. (2011), } \\
\text { Ferir et al. (2011), Kouokam et al. (2011), Xue et al. (2012), } \\
\text { Hoorelbeke et al. (2013), Nixon et al. (2013), Xue et al. (2013), } \\
\text { Barton et al. (2014), Moulaei et al. (2015), Barton et al. (2016), } \\
\text { Kouokam et al. (2016) }\end{array}$ \\
\hline & Anti-herpes simplex virus-2 & Nixon et al. (2013), Levendosky et al. (2015) \\
\hline & Anti-human papillomavirus & Levendosky et al. (2015) \\
\hline & Anti-hepatitis $\mathrm{C}$ virus & Meuleman et al. (2011), Takebe et al. (2013) \\
\hline & Anti-Japanese encephalitis activity & Ishag et al. (2013), Ishag et al. (2016) \\
\hline & $\begin{array}{l}\text { Anti-Middle East respiratory } \\
\text { syndrome coronavirus }\end{array}$ & Millet et al. (2016) \\
\hline & Anti-SARS coronavirus & O’Keefe et al. (2010) \\
\hline Halosaccion glandiforme & Anti-cancer & Perdhana (2017) \\
\hline \multirow[t]{3}{*}{ Hypnea cervicornis } & $\begin{array}{l}\text { Anti-nociceptive and anti-inflammatory } \\
\text { potential }\end{array}$ & Nascimento et al. (2006), Bitencourt et al. (2008) \\
\hline & Anti-hypernociceptive potential & Figueiredo et al. (2010) \\
\hline & Anti-cancer & Okuyama et al. (2009) \\
\hline H. musciformis & Anti-fungal & Melo et al. (1997) \\
\hline \multirow[t]{2}{*}{ Kappaphycus alvarezii } & Anti-influenza & Sato et al. (2011), Sato et al. (2015) \\
\hline & Anti-human immunodeficiency virus & Hirayama et al. (2016) \\
\hline Pterocladiella capillacea & Anti-nociceptive and anti-inflammatory & Silva et al. (2010), Abreu et al. (2012) \\
\hline \multirow[t]{3}{*}{ Solieria filiformis } & Anti-nociceptive and anti-inflammatory & Abreu et al. (2012), Abreu et al. (2016) \\
\hline & Antibacterial & Holanda et al. (2005) \\
\hline & Anti-cancer & Chaves et al. (2017) \\
\hline S. robusta & Mitogenic & Hori et al. (1988a) \\
\hline Tichocarpus crinitus & Mitogenic & Molchanova et al. (2010) \\
\hline
\end{tabular}

administration as it is readily bioavailable in rats after intravenous and subcutaneous treatments. They also suggested the multi-use of GRFT for treatment of systemic and enteric infections thus suggesting its role for anti-viral therapy and prevention of rectal transmission of HIV-1 and other susceptible viruses. Another lectin from red algae $K$. alvarezii (KAA) 
and its recombinant form (His-tagged rKAA-1) interacts with gp120 glycoprotein of HIV owing their high-mannose specificity and are able to inhibit its entry into host cell with an $\mathrm{IC}_{50}$ value of 7.3-12.9 nM (Hirayama et al. 2016).

\section{Anti-hepatitis virus}

Belonging to the Hepacivirus genus in the Flaviviridae family, hepatitis $\mathrm{C}$ virus (HCV) is a small positive single-stranded RNA virus which infects hepatocytes and causes serious liver diseases in humans (Simmonds, 2013). HCV chronically infects around 170 million people worldwide and about one third of them are at risk of developing liver fibrosis, cirrhosis and hepatocellular carcinoma in the coming decades (Dienstag and McHutchison 2006). HCV entry into hepatocytes is a complex multistep process that involves viral envelope glycoproteins along with several other cell entry factors (Dubuisson and Cosset 2014). Two heavily $N$-glycosylated envelope glycoproteins (E1 and E2) are present on the surface of viral particles and these $N$-glycans act as a promising target for new anti-viral strategies (Vieyres et al. 2010). GRFT specifically interacts with $N$-linked high-mannose oligosaccharides that are present on the viral envelope and can prevent $\mathrm{HCV}$ infection to hepatocytes in vitro and mitigate HCV infection in vivo (Meuleman et al. 2011). Thus, GRFT treatment has important clinical relevance as it prevents reinfection of the new liver graft which commonly occurs following liver transplantation in HCV-infected patients (Meuleman et al. 2011; Takebe et al. 2013).

\section{Anti-herpes simplex virus and anti-human papilloma virus}

Griffithsin (GRFT) is a promising broad-spectrum microbicide as it blocks herpes simplex virus 2 (HSV-2) infection and also inhibits human papilloma virus (HPV) (Levendosky et al. 2015). Mechanism of action of GRFT against HSV-2 involves entry inhibition (Nixon et al. 2013) along with prevention of cell-to-cell spread (Levendosky et al. 2015). Four glycoproteins $(\mathrm{gD}, \mathrm{gB}, \mathrm{gH}$ and $\mathrm{gL})$ play a role in viral entry and amongst them HSV gD is essential both for viral entry and for cell-to-cell spread and explains mechanism of action of GRFT (Levendosky et al. 2015). GRFT-gD binding occludes the receptor contact site of $\mathrm{gD}$, thus inhibits the critical role that gD plays in HSV-2 entry (Levendosky et al. 2015). Thus, viral entry inhibition in enveloped viruses by GRFT emphasises the role of $\mathrm{N}$-glycosylation in viral entry and the coincident susceptibility to anti-viral lectins as GRFT has high affinity for mannose-rich $N$-linked glycans (Levendosky et al. 2015). Further, GRFT also inhibits non-enveloped virus HPV by targeting $\alpha_{6}$ integrin (secondary receptor) or HPV-integrinGRFT complex is internalised without leading to infection thus suggesting involvement of secondary HPV receptor internalization (Levendosky et al. 2015). Thus, mechanism of action of GRFT against HSV2 is comparable to its action against HIV by interfering with virus entry and not involving attachment (Alexandre et al. 2011).

\section{Anti-influenza virus}

Various viral envelope glycoproteins are glycosylated, which play role in attachment and fusion of influenza viral envelope glycoprotein (haemagglutinin) to host endosomal membranes (Neumann and Kawaoka 2011). These influenza viruses cause annual epidemics or occasional global pandemics such as H1N1 subtypes which emerged in 2009 and spread pandemics all over world (Neumann and Kawaoka 2011). High-mannose binding lectin from $K$. alvarezii exhibits anti-influenza activity (Sato et al. 2011). Kappaphycus alvarezii (KAA-2) lectin inhibits influenza strains with $\mathrm{EC}_{50}$ of low nanomolar levels by interfering with virus entry into host cell (Sato et al. 2011). Owing to high-mannose binding specificity of ESA-2 lectin from $E$. serra, it inhibits influenza virus infection with an $\mathrm{EC}_{50}$ value of $12.4 \mathrm{nM}$ with no cytotoxicity up to $1000 \mathrm{nM}$ (Sato et al. 2015). ESA-2 lectin directly binds to HM glycans on envelope glycoprotein and thus inhibits influenza virus propagation (Sato et al. 2015).

\section{Anti-coronavirus}

Griffithsin acts as a potent inhibitor of SARS-CoV infection (in vitro and in vivo) with very low toxicity (O'Keefe et al. 2010). Middle East respiratory syndrome coronavirus (MERS-CoV) epidemic emerged in Arabian Peninsula in 2012 and is still ongoing with $35 \%$ of mortality rate (Zaki et al. 2012; Alhogbani 2016). Coronavirus are enveloped viruses having single-stranded, positive sense RNA genome and possess large number of spike proteins on their surface which mediate virus entry (binding and fusion) into host cell (Millet and Whittaker 2014). The MERS-CoV protein is highly glycosylated with 19 predicted glycosylation sites and thus acts as target for carbohydrate-binding proteins (lectins). Spike proteins mediate binding to the host cell surface along with fusion of the viral envelope with host cell membrane and griffithsin inhibits spike protein function during entry thus inhibits MERS-CoV infection at the entry step (Millet et al. 2016). Thus, owing to mannose specificity of griffithsin, it acts as potent anti-coronavirus candidate and inhibits in vitro MERS-CoV infection and production with minimal effect on cell viability (Millet et al. 2016).

\section{Anti-encephalitis virus}

Belonging to the genus of Flavivirus, Japanese encephalitis virus (JEV) is a mosquito-borne virus and a leading cause of high morbidity and mortality rate in Southeast Asia and the 
Western Pacific region (Chung et al. 2007). The JEV genome contains three structural genes: capsid protein $(\mathrm{C})$, premembrane (prM) and envelope (E) (Sumiyoshi et al. 1987). The E protein contains two potential glycosylation sites (Dutta et al. 2010), whereas prM contains only one glycosylation site (Kim et al. 2008). E protein is involved in virus attachment, fusion, penetration, cell tropism, virulence and attenuation (Lindenbach et al. 2007), whereas prM plays role in virus release and pathogenesis (Kim et al. 2008). Griffithsin (GRFT) is a broad-spectrum anti-viral protein and possesses anti-viral activity against Japanese encephalitis virus (JEV) infection (Ishag et al. 2013). The dimeric structure of GRFT having six binding sites is the underlying cause of its high affinity to interact with the viral glycosylated proteins and prevent its entry into cells (Ziolkowska et al. 2006). Its mechanism of action involves binding of GRFT to the JEV glycosylated viral proteins, specifically $\mathrm{E}$ and prM; however, increasing concentrations of mannose inhibit GRFT binding to JEV as revealed by in vitro experiments using Pull-down assay and Co-immunoprecipitation (CO-IP) assay (Ishag et al. 2016). Thus, the binding of GRFT to the glycosylated viral proteins contributes to its anti-JEV activity and has potentials in the development of therapeutics against JEV or other flavivirus infection (Ishag et al. 2016).

\section{Anti-nociceptive, anti-inflammatory and anti-hypernociceptive}

Lectin from A. multifida (LEC) has potential as an analgesic drug as it is a potent central and peripheral natural antinociceptive compound (Neves et al. 2007). Oral or intraperitoneal administration of lectin in Swiss mice inhibits acetic acid-induced abdominal writhings in dose-dependant manner (Neves et al. 2007). Anti-nociceptive action of LEC involves D-mannose as a pretreatment with this carbohydrate abolishing lectin activity (Neves et al. 2007). Pterocladiella capillacea lectin $(\mathrm{PcL})$ exhibits peripheral action with antinociceptive and anti-inflammatory activity (Silva et al. 2010). Intravenous administration of lectin (PcL) to Swiss mice significantly reduces writhings in a dose-dependant manner and reduces neutrophil migration in Wistar rats (Silva et al. 2010).

Solieria filiformis $(\mathrm{SfL})$ and $P$. capillacea $(\mathrm{PcL})$ lectins have anti-inflammatory effects (Abreu et al. 2012). SfL and PcL lectins are effective in controlling inflammatory processes as they are not cytotoxic, promotes increased cell viability and induces Th2 immune response in mouse spleenocytes (Abreu et al. 2012). Lectin from S. filiformis can also act as a therapeutic agent owing to its anti-nociceptive and anti-inflammatory potential (Abreu et al. 2016). Anti-nociceptive and antiinflammatory activity of $S$. filiformis lectin was evaluated in Swiss mice and Wistar rats, respectively, which showed reduced number of abdominal writhings, reduced paw licking times and reduced neutrophil migration in a peritonitis model along with no signs of systemic damage (Abreu et al. 2016).

Hypnea cervicornis agglutinin (HCA), along with antinociceptive and anti-inflammatory effects (Nascimento et al. 2006; Bitencourt et al. 2008), also exhibits antihypernociceptive effect in rats (Figueiredo et al. 2010). Intravenous administration of HCA to rats inhibits carrageenan and antigen-induced hypernociception, inhibits neutrophil migration and leads to an increase in nitric oxide production (Figueiredo et al. 2010). Further carbohydrate-binding sites play an important role as lectin effect was inhibited, when combined with mucin (Figueiredo et al. 2010).

\section{Anti-cancer}

Amongst all the malignant tumors, osteosarcoma has worst prognosis with low survival rate of treated patients (Link et al. 1991). Carbohydrate structures vary amongst normal and different cancer cell lines, with carcinomas and sarcomas having common surface bound sugar structures (Stastny and Gupta 1994). Specific interactions between unique sugar chains of high-mannose type on carcinoma cell surface and E. serra agglutinin lead to specific binding of agglutinin to carcinoma cells and induce apoptotic cell death (Sugahara et al. 2001). At concentration above $1.2 \mu \mathrm{g} \mathrm{mL}^{-1}$, ESA induces cell death against human colon cancer (Colo201) and human cervix cancer (HeLa cells) (Sugahara et al. 2001). Binding of E. serra agglutinin to sarcoma cells also induces apoptosis with higher anti-proliferative activity of lectin than in carcinoma cells (Hayashi et al. 2012).

Owing to exceptional carbohydrate specificity of red algal lectins, they can distinguish between membrane structures and act as a useful tool in typing of malignant cells (Shiomi et al. 1979; Kamiya et al. 1980; Kamiya et al. 1982; Pinto et al. 2009). Owing to unique carbohydrate specificity of Hypnin A-3 towards core ( $\alpha 1-6)$ fucosylated $N$-glycans, it might act as a potential candidate for cancer diagnosis as core ( $\alpha 1-6) \mathrm{fu}-$ cose is a critical marker for lung and liver cancer (Okuyama et al. 2009).

Lectin from B. seaforthii has potential as prognostic marker in primary central nervous system (CNS) tumor (Conrado et al. 2012). Owing to strict specificity of $B$. triquetrum lectin for the core $\alpha 1,6$-fucosylation, which is an important marker for cancerogenesis, rBTL is a potent candidate as a marker to detect core-fucosylation of animal cell surface glycoproteins and as cancer diagnosis tool (Nascimento et al. 2015). Halosaccion glandiforme lectin inhibits HeLa cervical cancer cell growth and have no cytotoxic effect against MCF-7 as well as HeLa cells (Perdhana 2017). Solieria filiformis isolectins have specificity towards high-mannose oligosaccharide present in the MCF-7 thus significantly inhibits 50\% 
of MCF-7 breast cancer cell viability at a concentration of $125 \mu \mathrm{g} \mathrm{mL}^{-1}$ (Chaves et al. 2017).

\section{Anti-microbial}

Various algal lectins can act as anti-microbial agents and interfere in biofilm formation owing to their carbohydrate specificity (Vasconcelos et al. 2014). Hypnea musciformis lectin exhibits anti-fungal activity against the dermatophyte fungus Trichophyton rubrum and plant pathogen Colletotrichum lindemuthianum (Melo et al. 1997). Purified lectins from E. serra and Galaxaura marginata has inhibitory activity against Vibrio vulnificus (Liao et al. 2003). Solieria filiformis lectin has an inhibitory effect over Gram-negative bacterial species such as Serratia marcescens, Salmonella typhi, Klebsiella pneumoniae, Enterobacter aerogenes, Proteus sp. and Pseudomonas aeruginosa (Holanda et al. 2005). Soliera filiformis lectin exhibits bacteriostatic effect owing to its interaction with glycoconjugate mannan or its hapten over surface walls of Gram-negative bacteria (Holanda et al. 2005). However, non-inhibitory effect of $S$. filiformis lectin over Gram-positive bacteria or some Gram-negative bacteria such as Escherichia coli and Salmonella typhimurium is due to non-accessibility of specific carbohydrates on cell wall of these bacteria or due to the absence of these carbohydrates (Holanda et al. 2005). Perdhana et al. (2017) determined the cytotoxicity and anti-bacterial effect of crude lectin fractions from red macro algae collected from the southern coast of Java Island, Indonesia. Crude protein extract from G. fisheri (GPE) has an anti-bacterial activity against Vibrio parahaemolyticus (Boonsri et al. 2017). Thus, owing to its anti-bacterial potential, G. fisheri could be used as a feed supplement in shrimp culture to protect against or prevent acute hepatopancreatic necrosis disease (Boonsri et al. 2017).

\section{Miscellaneous applications}

Owing to unique carbohydrate specificity of red algae lectins, which identify glycans attached to cell surfaces, glycoconjugates or free sugars, they can detect abnormal cells and biomarkers related to diseases and thus play a significant role in treatments of various immunological diseases, wounds, etc. Selective adsorption of salivary molecules to the teeth leads to formation of an organic and acellular film known as acquired enamel pellicle (Yin et al. 2005). Dental caries involve bacterial adhesion to this acquired pellicle as initial stage of plaque formation (Scheie 1994). Thus, an effective solution towards this problem involves anti-adhesion lectin therapy which interferes with bacterial adhesion and aggregation (Teixeira et al. 2006). Through competitive mechanism, B. seaforthii lectin (BSL) blocks oral streptococci adhesion to their mucin receptors in acquired pellicle and presents great stability (Teixeira et al. 2007).
A major problem in public health in many countries is disruption of skin integrity which leads to cutaneous wounds (Schreml et al. 2010; Chen et al. 2012). Highly specialised cells interact with extracellular matrix during complex healing process which leads to growth and tissue repair (Diegelmann and Evans, 2004; Goldberg and Diegelmann, 2010). Owing to potential of lectins for promoting healing, they act as a promising candidate in treatment of skin wounds (Chahud et al. 2009; Nascimento Neto et al. 2011). A lectin from B. seaforthii has a pro-healing activity as it accelerates healing of skin wounds by exerting activity on migration of polymorphonuclear cells towards injured site (Nascimento-Neto et al. 2012).

Lectin from $G$. cornea has acaricidal activity as exposure of lectin to female cattle tick (Boophilus microplus) significantly reduces cattle tick weight after the oviposition period, egg mass weight, hatching period and mean larvae survival time (Lima et al. 2005).

Carpopeltis flabellata (Hori et al. 1987), S. robusta (Hori et al. 1988a) and G. bursa-pastoris (Okamoto et al. 1990) lectins exhibit mitogenic potential towards mouse splenic lymphocytes from BALB/c mice. Eucheuma serra lectins (ESA-1 and ESA-2) stimulate mitogenesis in mouse lymphocytes (Kawakubo et al. 1997). Gracilaria tikvahiae (HBOI Strain G5) and G. verrucosa (HBOI Strain G-16S) lectins are highly mitogenic for murine splenocytes; however, G. tikvahiae (McLachlan HBOI Strain G-3) lectin is mitogenic for human lymphocytes (Bird et al. 1993). Tichocarpus crinitus lectin also has a weak mitogenic effect on human lymphocytes in vitro and has the ability to stimulate synthesis of pro-inflammatory cytokines by human whole blood cells (Molchanova et al. 2010).

\section{Conclusions}

Robust preventive measures are required to deal with worldwide spread of enveloped viruses such as HIV, HSV, HCV and SARS-CoV. Owing to carbohydrate specificity of red algae lectins towards glycoproteins present on enveloped viruses and their broad-spectrum activity against susceptible viruses, these lectins are being advocated for further development as anti-viral agents. Lectins from red algae have specificity towards complex glycoproteins or high-mannose glycans with a few lectins having affinity towards monosaccharides. Owing to their sugar specificity, further their agglutination preferences are understood. Thus, based on their carbohydrate specificity, red algae lectins have potential biomedical application like anti-viral, anti-cancer and anti-microbial agents. Owing to anti-nociceptive and anti-inflammatory effects of red algae lectins, they act as promising tools to discover safe and efficient agents for pain therapy. However, owing to lectin stability and toxicity concerns, there has been limited clinical translation of major research findings conducted in lectinology field. 
Acknowledgements Financial support in the form of Maulana Azad National Fellowship from University Grants Commission, New Delhi, Government of India is gratefully acknowledged by A.K. Walia.

\section{Compliance with ethical standards}

Conflict of interest Authors declare that they have no conflict of interest.

\section{References}

Abreu TM, Ribeiro NA, Chaves HV, Jorge RJ, Bezerra MM, Monteiro HS, Vasconcelos IM, Mota ÉF, Benevides NM (2016) Antinociceptive and anti-inflammatory activities of the lectin from marine red alga Solieria filiformis. Planta Med 82:596-605

Abreu TM, Silva LMCM, Vanderlei ESO, de Melo CML, Pereira VRA, Benevides NMB (2012) Cytokine production induced by marine algae lectins in BALB/c mice splenocytes. Protein Pept Lett 19: 975-981

Ainouz IL, Sampaio AH, Benevides NMB, Feritas ALP, Costa FHF, Carvalho MR, Pinheiro-Joventino F (1992) Agglutination of enzyme treated erythrocytes by Brazalian marine algal extract. Bot Mar 35:475-479

Ainouz IL, Sampaio AH, Freitas ALP, Benevides NMBB, Mapurunga S (1995) Comparative study on hemagglutinins from the red algae Bryothamnion seaforthii and Bryothamnion triquetrum. R Bras Fisiol Veg 7:15-19

Ainouz L, Sampaio H (1991) Screening of Brazilian marine algae for hemagglutinins. Bot Marina 34:211-214

Akkouh O, Ng TB, Singh SS, Yin C, Dan X, Chan YS, Pan W, Cheung RC (2015) Lectins with anti-HIV activity: a review. Molecules 20: 648-668

Alexandre KB, Gray ES, Pantophlet R, Moore PL, McMahon JB, Chakauya E, O'Keefe BR, Chikwamba R, Morris L (2011) Binding of the mannose-specific lectin, griffithsin, to HIV-1 gp120 exposes the CD4-binding site. J Virol 85:9039-9050

Alhogbani T (2016) Acute myocarditis associated with novel Middle East respiratory syndrome coronavirus. Ann Saudi Med 36:78-80

Ambrosi M, Cameron NR, Davis BG (2005) Lectins: tools for the molecular understanding of the glycocode. Org Biomol Chem 3:15931608

Anam C, Praseptiangga D, Nugraheni MA, Nurhayati T, Fajarningsih ND, Zilda DS, Chasanah E, Yunus A (2017a) Preliminary characterization of crude lectin fraction of the red alga, Acrocystis nana from wediombo beach of the southern coast of Java island, Gunung kidul, Yogyakarta, Indonesia. IOP Conf Ser: Mater Sci Eng 193: 012016

Anam C, Chasanah E, Perdhana BP, Fajarningsih ND, Yusro NF, Sari AM, Nursiwi A, Praseptiangga D, Yunus A (2017b) Cytotoxicity of crude lectins from red macroalgae from the southern coast of Java island, Gunung Kidul Regency, Yogyakarta, Indonesia. IOP Conf Ser: Mater Sci Eng 193:012017

Barton C, Kouokam JC, Lasnik AB, Foreman O, Cambon A, Brock G, Montefiori DC, Vojdani F, McCormick AA, O'Keefe BR, Palmer KE (2014) Activity of and effect of subcutaneous treatment with the broad-spectrum antiviral lectin griffithsin in two lab rodent models. Antimicrob Agents Chemother 58:120-127

Barton C, Kouokam JC, Hurst H, Palmer KE (2016) Pharmacokinetics of the antiviral lectin griffithsin administered by different routes indicates multiple potential uses. Viruses 8:12

Benevides NMB, Holanda ML, Melo FR, Fieitas ALP, Sampaio AH (1998) Purification and partial characterization of the lectin from marine red alga Enatiocladia duperreyi (C. Agardh) Falkenberg. Bot Mar 41:521-525

Benevides NMB, Leite AM, Freitas ALP (1996) Atividade hemaglutinante na alga vermelha Solieria filiformis. R Bras Fisiol Veg 8:117-122

Bies C, Lehr C, Woodley JF (2004) Lectin-mediated drug targeting: history and applications. Adv Drug Deliv Rev 56:402-435

Bird KT, Chiles TC, Longley RE, Kendrick AF, Kinkema MD (1993) Agglutinins from marine macroalgae of the south eastern United States. J Appl Phycol 5:213-218

Bitencourt F, da S, Figueiredo JG, Mota MRL, Bezerra CCR, Silvestre PP, Vale MR, Nascimento KS, Sampaio AH, Nagano CS, SakerSampaio S, Farias WRL, Cavada BS, Assreuy AMS, de Alencar NMN (2008) Antinociceptive and anti-inflammatory effects of a mucin-binding agglutinin isolated from the red marine alga Hypnea cervicornis. Naunyn Schmiedeberg's Arch Pharmacol 377:139-148

Blunden G, Rogers DJ, Farnham WF (1975) Survey of British seaweeds for hemagglutinins. Lloydia 38:162-168

Boonsri N, Rudtanatip T, Withyachumnarnkul B, Wongprasert K (2017) Protein extract from red seaweed Gracilaria fisheri prevents acute hepatopancreatic necrosis disease (AHPND) infection in shrimp. J Appl Phycol 29:1597-1608

Boyd WC, Almodovar LR, Boyd LG (1966) Agglutinins in marine algae for human erythrocytes. Transfusion 6:82-83

Cabezas JA (1973) The type of naturally occurring sialic acids. Rev Esp Fisiol 29:307-322

Cabezas M, Cabezas JA (1973) Comparative study on the composition of platelets from the equine, bovine, ovine and porcine species. Rev Esp Fisiol 29:323-328

Calvete JJ, Costa FHF, Saker-Sampio S, Murciano MPM, Nagano CS, Cavada BS, Grangeiro TB, Ramos MV, Jr CB, Silveira SB, Freitas BT, Sampio AH (2000) The amino acid sequence of the agglutinin isolated from the red marine algae Bryothamnion triquetrum defines a novel lectin structure. Cell Mol Life Sci 57:343-350

Chahud F, Ramalho LNZ, Ramalho FS, Haddad A, Roque-Barreira MC (2009) The lectin KM+ induces corneal epithelial wound healing in rabbits. Int J Exp Pathol 90:166-173

Chaves RP, da Silva SR, Nascimento Neto LG, Carneiro RF, Coelho da Silva AL, Sampaio AH, lopes de Sousa B, Cabral MG, Videira PA, Teixeira EH, Nagano CS (2017) Structural characterization of two isolectins from the marine red alga Solieria filiformis (Kützing) P.W. Gabrielson and their anticancer effect on MCF-7 breast cancer cells. Int J Biol Macromol. https://doi.org/10.1016/j.ijbiomac.2017.09. 116

Chen X, Peng LH, Li N, Li QM, Li P, Fung KP, Leung PC, Gao JQ (2012) The healing and anti-scar effects of astragaloside IV on the wound repair in vitro and in vivo. J Ethnopharmacol 139:721-727

Chernikov OV, Chikalovets IV, Molchanova VI, Pavlova MA, Lukyanova PA (2007) Algae of Peter the great bay of the sea of Japan as a source of lectins. Russ J Mar Biol 33:329-332

Cheung RCF, Wong JH, Pan W, Chan YS, Yin C, Dan X, Ng TB (2015) Marine lectins and their medicinal applications. Appl Microbiol Biotechnol 99:3755-3773

Chiles TC, Bird KT (1989) A comparative study of animal erythrocyte agglutinins from marine algae. Comp Biochem Physiol 94:107-111

Chiles TC, Bird KT (1990) Gracilaria tikvahiae agglutinin. Partial purification and preliminary characterization of its carbohydrate specificity. Carbohydr Res 207:319-326

Chung C-C, Lee S-J, Chen Y-S, Tsai H-C, Wann S-R, Kao C-H, Liu Y-C (2007) Acute flaccid paralysis as an unusual presenting symptom of Japanese encephalitis: a case report and review of the literature. Infection 35:30-32

Conrado FM, Furtado LETA, Teixeira AH, Coutinho NLP, Sampaio AH, Cavada BS, Bezerra MM, Silva AAR, Barbosa FCBB, Chaves HV, Filho GC, Pinto VPT (2012) Erythrina velutina and Bryothamnion 
seaforthii lectins binding to proteins of primary central nervous system tumors. J Cancer Res Exp Oncol 41:21-26

Cunliffe DA, Cox KO (1979) Effects of bromelain and pronase on erythrocyte membranes. Mol Immunol 16:427-433

De Souza BWS, Teixeria DIA, Andrade FK, Melo MRS, Munzo AM, Freitas ALP (2007) A survey of antartic algae for agglutinins. Oceol Bras 11:122-130

Diegelmann RF, Evans MC (2004) Wound healing: an overview of acute, fibrotic and delayed healing. Front Biosci 9:283-289

Dienstag JL, McHutchison JG (2006) American gastroenterological association technical review on the management of hepatitis C. Gastroenterol 130:231-264

Dinh HL, Hori K, Quang NH (2009) Screening and preliminary characterization of hemagglutinins in Vietnamese marine algae. J Appl Phycol 21:89-97

Dixion HBF (1981) Defining a lectin. Nature 292:192

Drickamer K (1988) Two distinct classes of carbohydrate-recognition domains in animal lectins. J Biol Chem 263:9557-9560

Dubuisson J, Cosset FL (2014) Virology and cell biology of the hepatitis C virus life cycle - an update. J Hepatol 61:S3-S13

Dutta K, Rangarajan PN, Vrati S, Basu A (2010) Japanese encephalitis: pathogenesis, prophylactics and therapeutics. Curr Sci 98:326-334

Emau P, Tina B, O'feeke BK, Mori T, McMohan JB, Palmer KC, Jiang Y, Bekele G, Tsai CC (2007) Griffithsin, a potent HIV entry inhibitor, is an excellent candidate for anti-HIV microbicide. J Med Primatol 36: 244-253

Eylar EH, Madoff MA, Brody OV, Oncley JL (1962) The contribution of sialic acid to the surface charge of the erythrocyte. J Biol Chem 237: 1992-2000

Fabregas J, Lopez A, Llovo J, Munoz A (1992) A comparative study of seafish erythrocytes and agglutinins from seaweeds. Comp Biochem Physiol A 103:307-313

Ferir G, Palmer KE, Schols D (2011) Synergistic activity profile of griffithsin in combination with tenofovir, maravirus \& enfuvirtide against HIV-1 clade C. Virology 47:253-258

Figueiredo JG, Bitencourt FS, Cunha TH, Luz PB, Nascimento KS, Mota MRL, Sampaio AH, Cavada BS, Cunha FQ, Alencar NMN (2010) Agglutinin isolated from the red marine alga Hypnea cervicornis $\mathbf{J}$ Agardh reduces inflammatory hypernociception: involvement of nitric oxide. Pharmacol Biochem Behav 96:371-377

Fukuda Y, Sugahara T, Ueno M, Fukuta Y, Ochi Y, Akiyama K, Miyazaki T, Masuda S, Kawakubo A, Kato K (2006) The anti-tumor effect of Euchema serra agglutinin on colon cancer cells in vitro and in vivo. Anticancer Drugs 17:943-947

Fuqua JL, Hamorsky K, Khalsa G, Matoba N, Palmer KE (2015) Bulk production of the antiviral lectin griffithsin. Plant Biotech J 13: $1160-1168$

Furthmayr H (1977) Structural analysis of a membrane glycoprotein: glycophorin A. J Supramol Struc 7:121-134

Goldberg SR, Diegelmann RF (2010) Wound healing primer. Surg Clin N Am 90:1133-1146

Hara T, Aramaki Y, Takada S, Koike S, Tsuchiya S (1995) Receptormediated transfer of pSV2CAT DNA to mouse liver cells using asialofetuin-labeled liposomes. Gene Ther 2:784-788

Hayashi K, Walde P, Miyazaki T, Sakayama K, Nakamura A, Kameda K, Masuda S, Umakoshi H, Kato K (2012) Active targeting to osteosarcoma cells and apoptotic cell death induction by the novel lectin Eucheuma serra agglutinin isolated from a marine red alga. J Drug Del 2012:842785

Hirayama M, Shibata H, Imamura K, Takemasa Sakaguchi T, Hori K (2016) High-mannose specific lectin and its recombinants from a carrageenophyta Kappaphycus alvarezii represent a potent anti-HIV activity through high-affinity binding to the viral envelope glycoprotein gp120. Mar Biotechnol 18:144-160

Holanda ML, Melo VMM, Silva LMCM, Amorim RCN, Pereira MG, Benevides NMB (2005) Differential activity of a lectin from Solieria filiformis against human pathogenic bacteria. Braz J Med Biol Res 38:1769-1773

Hoorelbeke B, Xue J, LiWang PJ, Balzarini J (2013) Role of the carbohydrate-binding sites of griffithsin in the prevention of DCSIGN-mediated capture and transmission of HIV-1. PLoS One 8: e64132

Hori K, Ikegam S, Miyazawa K, Ito k (1988a) Mitogenic and antineoplastic isoagglutinins from the red alga Soleria robusta. Phytochemistry 27:2063-2067

Hori K, Matsubara K, Miyazawa K (2000) Primary structures of two hemagglutinins from the marine red alga, Hypnea japonica. Biochim Biophys Acta 1474:226-236

Hori K, Miyazawa K, Fusetani N, Hashimoto K, Ito K (1986b) Hypnins, low molecular weight peptidic agglutinins from a marine red alga, Hypnea japonica. Biochim Biophys Acta 873:228-236

Hori K, Miyazawa K, Ito K (1986a) Preliminary characrerization of agglutinins from seven marine algal species. Bull J Soc Sci Fish 52:323-331

Hori K, Miyazawa K, Ito K (1987) A mitogenic agglutinin from the red alga Carpopeltis flabellata. Phytochemistry 26:1335-1338

Hori K, Miyazawa K, Ito K (1990) Some common properties of lectins from marine algae. Hydrobiologia 204-205:561-566

Hori K, Ogata T, Kamiya H, Mimuro M (1996) Lectin like compounds and lectin receptors in marine microalgae hemagglutination and reactivity with purified lectins. J Phycol 32:783-790

Hori K, Oiwa C, Miyazawa K, Ito K (1988b) Evidence for wide distribution of agglutinins in marine algae. Bot Mar 31:133-138

Hori K, Sato Y, Ito K, Fujiwara Y, Iwamoto Y, Makino H, Kawakubo A (2007) Strict specificity for high-mannose type $\mathrm{N}$-glycans and primary structure of a red alga Eucheuma serra lectin. Glycobiol 17:479-491

Hung LD, Hirayama M, Ly BM, Hori K (2015) Purification, primary structure, and biological activity of the high-mannose N-glycanspecific lectin from cultivated Eucheuma denticulatum. J Appl Phycol 27:1657-1669

Hung LD, Hori K, Nang HQ, Kha T, Hoa LT (2009) Seasonal changes in growth rate, carrageenan yield and lectin content in red alga Kappaphycus alvarezii cultivated in Camrah Bay, Vietnam. J Appl Phycol 21:265-272

Hung LD, Sato Y, Hori K (2011) High-mannose N-glycan-specific lectin from the red alga Kappaphycus striatum (Carrageenophyte). Phytochemistry 72:855-861

Ishag HZ, Li C, Wang F, Mao X (2016) Griffithsin binds to the glycosylated proteins (E and prM) of Japanese encephalitis virus and inhibit its infection. Virus Res 215:50-54

Ishag HZF, Li C, Li H, Ming-Xia S, Wang F, Bo N, Matik T, Pu-Yan C, Mao X (2013) Griffithsin inhibits Japanese encephalitis virus infection in vitro and in vivo. Arch Virol 158:349-358

Kakita H, Fukuoka S, Obika H, Kamishima H (1999) Isolation and characterisation of a fourth hemagglutinin from the red alga, Gracilaria verrucosa, from Japan. J Appl Phycol 11:49-56

Kakita H, Fukuoka S, Obika H, Li ZR, Kamishima H (1997) Purification and properties of a high molecular weight hemagglutinin from the red alga, Gracilaria verrucosa. Bot Mar 40:241-247

Kamiya H, Ogata K, Hori K (1982) Isolation and characterization of a new agglutinin in the red alga Palmaria palmata (L) O Kuntze. Bot Mar 25:537-540

Kamiya H, Shiomi K, Shimizu Y (1980) Marine biopolymers with cell specificity-III in the red algae Cystoclonium purpureum: isolation and characterization. J Nat Prod 43:136-139

Kawakubo A, Makino H, Ohnishi J, Hirohara H, Kanji H (1997) The marine alga Eucheuma serra $\mathrm{J}$ Agardh, a high yielding source of two isolectins. J Appl Phycol 9:331-338

Kawakubo A, Makino H, Ohnishi J, Hirohara H, Kanji H (1999) Occurrence of highly yielded lectins homologous within genus Eucheuma. J Appl Phycol 11:149-156

Khan F, Khan RH, Sherwani A, Mohmood S, Azfer MA (2002) Lectins as markers for blood grouping. Med Sci Monit 8:RA293-RA300 
Kim JM, Yun SI, Song BH, Hahn YS, Lee CH, Oh HW, Lee YM (2008) A single N-linked glycosylation site in the Japanese encephalitis virus prM protein is critical for cell type-specific prM protein biogenesis, virus particle release, and pathogenicity in mice. J Virol 82: 7846-7862

Kouokam JC, Huskens D, Schols D, Johannemann A, Riedell SK, Walter W, Walker JM, Matobal N, O'Keefe BR, Palmer KE (2011) Investigation of Griffithsin's interactions with human cells confirms its outstanding safety and efficacy profile as a microbicide candidate. PLoS One 6: e22635

Kouokam JC, Lasnik AB, Palmer KE (2016) Studies in a murine model confirm the safety of Griffithsin and advocate its further development as a microbicide targeting HIV-1 and other enveloped viruses. Viruses 8:311

Lambert R, Edwards J, Anstee DJ (1977) A simple method for the standardisation of proteolytic enzymes used in blood group serology. Med Lab Sci 35:233-238

Le HD, Sato T, Shibata H, Hori K (2009) Biochemical comparison of lectins among three different color strains of the red alga Kappaphycus alvarezii. Fisheries Sci 75:723-730

Leite YFMM, Silva LMCM, Amorim RCNA, Freire EA, Jorge DMM, Grangeiro TB, Benevides NMB (2005) Purification of a lectin from the marine red alga Gracilaria ornata and its effect on the development of the cowpea weevil Callosobruchus maculates (Coleoptera: Bruchidae). Biochim Biophys Acta 1724:137-145

Levendosky K, Mizenina O, Martinelli E, Jean-Pierre N, Kizima L, Rodriguez A, Kleinbeck K, Bonnaire T, Robbiani M, Zydowsky TM, O'Keefe BR, Fernández-Romero JA (2015) Griffithsin and carrageenan combination to target HSV-2 and HPV. Antimicrob Agents Chemother 59:7290-7298

Liao WR, Lin JY, Shieh WY, Jeng WL, Huang R (2003) Antibiotic activity of lectins from marine algae against marine vibrios. J Ind Microbiol Biotech 30:433-439

Liener IE, Sharon N, Goldstein IJ (1986) The lectins: properties, functions, and applications in biology and medicine. Academic Press, London

Lima MEP, Carneiro ME, Nascimento AE, Grangeiro TB, Holanda ML, Amorim RCN, Benevides NMB (2005) Purification of a lectin from the marine red alga Gracilaria cornea and its effects on the cattle tick Boophilus microplus (Acari: Ixodidae). J Agric Food Chem 53: 6414-6419

Lindenbach BD, Thiel H-JU, Rice CM (2007) Flaviviridae: the viruses and their replication. In: Knipe DM, Howley PM (eds) Fields virology. Lippincott-Raven, Philadelphia, pp 1108-1109

Link MP, Goorin AM, Horowitz M, Meyer WH, Belasco J, Baker A, Ayala A, Shuster J (1991) Adjuvant chemotherapy of high-grade osteosarcoma of the extremity: updated results of the multiinstitutional osteosarcoma study. Clin Orthop Relat Res 270:8-14

Lusvarghi S, Bewley CA (2016) Griffithsin: an antiviral lectin with outstanding therapeutic potential. Viruses 8:E296

Marchesi VT, Furthmayr H, Tomita M (1976) The red cell membrane. Annu Rev Biochem 45:667-698

Matsubara K, Sumi H, Hori K (1996) Platelet aggregation is inhibited by phycolectins. Experientia 52:540-543

Melo FR, Norma MB, Benevides NMB, Pereira MG, Holanda ML, Mendes FNP, Oliveira SRM, Freitas LP, Silva LMCM (2004) Purification and partial characterisation of a lectin from the red marine alga Vidalia obtusiloba C Agardh. Rev Bras Bot 27:263-269

Melo VMM, Medeiros DA, Rios EJB, Castelar LLM, Carvalho A de FFU (1997) Antifungal properties of proteins (agglutinins) from the red alga Hypnea musciformis (Wulfen) Lamouroux. Bot Mar 40:281-284

Meuleman P, Albecka A, Belouzard S, Vercauteren K, Verhoye L, Wychowski C, Roels GL, Palmer KE, Dubuisson J (2011) Griffithsin has antiviral activity against hepatitis $\mathrm{C}$ virus. Antimicrob Agents Chemother 55:5159-5162
Micevicz ED, Cole AL, Jung CL, Luong H, Phillips ML, Pratikhya P, Sharma S, Waring AJ, Cole AM, Ruchala P (2010) Grifonin-1: a small HIV-1 entry inhibitor derived from the algal lectin, Griffithsin. PLoS One 5:e14360

Millet JK, Whittaker GR (2014) Host cell entry of Middle East respiratory syndrome coronavirus after two-step, furin-mediated activation of the spike protein. PNAS 111:15214-15219

Millet JK, Seron K, Labitt RN, Danneels A, Palmer KE, Whittaker GR, Dubuisson J, Belouzard S (2016) Middle East respiratory syndrome coronavirus infection is inhibited by griffithsin. Antivir Res 133:1-8

Mirelman D (1986) Microbial lectins and agglutinins: properties and biological activity. John Wiley \& Sons, New York

Mo H, Goldstein IJ (1994) Isolation and characterization of a Forssman antigen-binding lectin from velvet bean (Mucuna derrigiana) seeds. Glycoconj J 11:424-431

Molchanova V, Chernikov O, Chikalovets I, Lukyanov P (2010) Purification and partial characterization of the lectin from the marine alga Tichocarpus crinitus (Gmelin) Rupr (Rhodophyta). Bot Mar 53:69-78

Mori T, O'Keefe BR, Sowder RC, Bringans S, Gardella R, Berg S, Cochran P, Turpin JA, Buckheit RW, McMahon JB, Boyd MR (2005) Isolation and characterization of griffithsin, a novel HIVinactivating protein, from the red alga Griffithsia spp. J Biol Chem 280:9345-9353

Moulaei T, Alexandre KB, Shenoy SR, Meyerson JR, Krumpe LR, Constantine B, Wilson J, Buckheit RW Jr, McMahon JB, Subramaniam S, Wlodawer A, O'Keefe BR (2015) Griffithsin tandemers: flexible and potent lectin inhibitors of the human immunodeficiency virus. Retrovirology 12:6

Moulaei T, Shenoy SR, Giomarelli B, Thomas C, McMahon JB, Dauter Z, O'Keefe BR, Wlodawer A (2010) Monomerization of viral entry inhibitor griffithsin elucidates the relationship between multivalent binding to carbohydrates and anti-HIV activity. Structure 18:11041115

Nagano CS, del Sol FG, Cavada BS, Nascimento KSD, Nunes EV, Sampaio AH, Calvetea JJ (2005) Crystallization and preliminary $\mathrm{X}$-ray diffraction analysis of HML, a lectin from the red marine alga Hypnea musciformis. Acta Cryst F 61:997-999

Nagano CS, Moreno FBMB, Bloch C, Prates MV, Calvete JJ, Sampaio SS, Farias WRL, Tavares TD, Nascimento KS, Grangeiro TB, Cavada BS, Sampaio AH (2002) Purification and characterization of a new lectin from red marine alga Hypnea musciformis. Protein Pept Lett 9:159-165

Nascimento KS, Nagano CS, Nunes EV, Rodrigues GV, Calvete JJ, Saker S, Sampaio WRL, Farias SAH (2006) Isolation and characterization of a new agglutinin from red marine alga Hypnea cervicornis $\mathrm{J}$ Agardh. Biochem Cell Biol 84:49-54

Nascimento ASF, Serena S, Beloqui A, Arda A, Sampaio AH, Walcher J, Ott D, Unverzagt G, Reichardt N-C, Jimenez-Barbero J, Nascimento KS, Imberty A, Cavada BS, Varrot A (2015) Algal lectin binding to core $(\alpha 1-6)$ fucosylated N-glycans: structural basis for specificity and production of recombinant protein. Glycobiol 6:607-616

Nascimento-Neto LG, Carneiro RF, da Silva SR, da Silva BR, Arruda FVS, Carneiro VA, do Nascimento KS, Saker-Sampaio S, da Silva VA Jr, Porto ALF, Cavada BS, Sampaio AH, Teixeira EH, Nagano CS (2012) Characterization of isoforms of the lectin isolated from the red algae Bryothamnion seaforthii and its pro-healing effect. Mar Drugs 10:1936-1954

Nascimento-Neto LG, Pinto LS, Bastos RM, Evaristo FFV, Vasconcelos MA, Carneiro VA, Arruda FVS, Porto ALF, Leal RB, Júnior VA, Cavada BS, Teixeira EH (2011) Effect of lectin from Bauhinia variegata and its recombinant isoform on surgically induced skin wounds in a murine model. Molecules 16:9298-9315

Neumann G, Kawaoka Y (2011) The first influenza pandemic of the new millennium. Influenza Other Respir Viruses 5:157-166 
Neves SA, Freitas ALP, Souza BWS, Rocha MLA, Correia1 MVO, Sampaio DA, Viana GSB (2007) Antinociceptive properties in mice of a lectin isolated from the marine alga Amansia multifida Lamouroux. Braz J Med Biol Res 40:127-134

Nixon B, Stenfanidor M, Mesquitai MM, Fakioglu E, Segarra T, Rohan L, Halford W, Palmer KE, Herold BC (2013) Griffithsin protects mice from genital herpes by preventing cell-to-cell spread. J Virol 87:6257-6269

O'Keefe BR, Giomarelli B, Barnard DL, Shenoy SR, Chan PKS, McMahon JB, Palmer KE, Barnett BW, Meyerholz DK, Wohlford-Lenane CL, McCray PB (2010) Broad-spectrum in vitro activity and in vivo efficacy of the antiviral protein Griffithsin against emerging viruses of the family Coronaviridae. J Virol 84: 2511-2521

O'Keefe BR, Vojdani F, Buffa V, Shattock RJ, Montefiori DC, Bakke J, Mirsalis J, d'Andrea Anna-Lisa, Hume SD, Bratcher B, Saucedo CJ, McMohan, JB, Pogue GP, Palmer KE (2009) Scaleable manufacture of HIV-1 entry inhibitor griffithsin and validation of its safety and efficacy as a topical microbicide component. PNAS 106:6099-6104

Okamoto R, Hori K, Miyazawa K, Ito K (1990) Isolation and characterization of a new hemagglutinin from the red alga Gracilaria bursapastoris. Experientia 46:975-977

Okamura M, Yokoyama N, Takabatake N, Okubo K, Ikehara Y, Igarashi I (2007) Modification of host erythrocyte membranes by trypsin and chymotrypsin treatments and effects on the in vitro growth of bovine and equine Babesia parasites. J Parasitol 93:208-211

Okuyama S, Nakamura-Tsuruta S, Tateno H, Hirabayashi J, Matsubara K, Hori K (2009) Strict binding specificity of small-sized lectins from the red marine alga Hypnea japonica for core $(\alpha-1,6)$ fucosylated N-glycans. Biosci Biotechnol Biochem 73:912-920

Oliveira SRM, Nascimento AE, Lima MEP, Leite YFMM, Benevides NMB (2002) Purification and characterisation of a lectin from the red marine alga Pterocladiella capillacea (SG Gmel) Santel \& Hommers. Rev Brasil Bot 25:397-403

Perdhana BP (2017) Cytotoxicity and antibacterial effects of crude lectin fraction bioactive compound of red macroalgae from the Southern Coast of Java island, Gunungkidul regency. Dissertation, Sebelas Maret University, Surakarta, Yogyakarta

Pfeil R, Kamerling JP, Kuster JM, Schauer R (1980) O-acetylated sialic acids in erythrocyte membranes of different species. Hoppe-Seylers Z Physiol Chem 361:314-315

Pinto VPT, Debray H, Dus D, Teixeira EH, de Oliveira TM, Carneiro VA, Teixeira AH, Filho GC, Nagano CS, Nascimento KN, Alexandre H, Sampaio AH, Cavada BS (2009) Lectins from the red marine algal species Bryothamnion seaforthii and Bryothamnion triquetrum as tools to differentiate human colon carcinoma cells. Adv Pharmacol Sci 2009:862162

Praseptiangga D (2015) Algal lectins and their potential uses. Squalen Bull Mar Fish Postharvest Biotech 10:89-98

Praseptiangga D (2017) Development of seaweed-based biopolymers for edible films and lectins. IOP Conf Ser: Mater Sci Eng 193:012003

Rabelo L, Monteiro N, Serquiz R, Santos P, Oliveira R, Oliveira A, Rocha H, Morais AH, Uchoa A, Santos E (2012) A lactose-binding lectin from the marine sponge Cinachyrella apion $(\mathrm{Cal})$ induces cell death in human cervical adenocarcinoma cells. Mar Drugs 10:727-743

Reuter G, Vliegenthart JFG, Wember M, Schauer R, Howard RJ (1980) Identification of 9-O-acetyl-N-acetylneuraminic acid on the surface of BALB/c mouse erythrocytes. Biochem Biophys Res Commun 94:567-572

Rogers DJ, Fish B, Barwell CJ (1990) Isolation and properties of lectins from two red marine algae: Plumaria elegans and Ptilota serrata. In: BogHansen TC, Freed DLJ (eds) Lectins: biology, biochemistry, clinical biochemistry. St. Louis, Sigma Chemical Company, pp 49-52

Rogers DJ, Hori K (1993) Marine algal lectins: new developments. Hydrobiologia 260/261:589-593
Rogers DJ, Topliss JA (1983) Purification and characterisation of an antisialic acid agglutinin from the red alga Solieria chordalis (C. Ag.) J. Ag. Bot Mar 16:301-305

Rüdiger H, Gabius HJ (2001) Plant lectins: occurrence, biochemistry, functions and applications. Glycoconj J 18:589-613

Sampaio AH, Rogers DJ, Barwell CJ (1998) A galactose-specific lectin from the red alga Ptilota filicina. Phytochemistry 48:765-769

Sampaio AH, Rogers DJ, Barwell CJ, Saker-Sampaio S, Costa FHF, Ramos MV (1999) A new isolation procedure and further characterisation of the lectin from the red marine alga Ptilota serrata. J Appl Phycol 10:539-546

Sampaio AH, Rogers DJ, Barwell CJ, Saker-Sampaio S, Nascimento KS, Nagano CS, Farias WRL (2002) New affinity procedure for the isolation and further characterization of the blood group B specific lectin from the red marine alga Ptilota plumosa. J Appl Phycol 14:489-495

Sato Y, Morimoto K, Hirayama M, Hori K (2011) High mannose-specific lectin (KAA-2) from the red alga Kappaphycus alvarezii potently inhibits influenza virus infection in a strain-independent manner. Biochem Biophys Res Commun 405:291-296

Sato Y, Morimoto K, Kubo T, Sakaguchi T, Nishizono A, Hirayama M, Hori K (2015) Entry inhibition of influenza viruses with high mannose binding lectin ESA-2 from red alga Eucheuma serra through the recognition of viral hemagglutnin. Mar Drugs 13:3454-3465

Scheie AA (1994) Mechanisms of dental plaque formation. Adv Dent Res 8:246-253

Schreml S, Szeimies R-M, Prantl L, Landthaler M, Babilas P (2010) Wound healing in the 21st century. J Am Acad Dermatol 63:866-881

Sharon N, Lis H (1972) Lectins: cell agglutinating and sugar specific proteins. Science 177:949-959

Sharon N, Lis H (2004) History of lectins: from hemagglutinins to biological recognition molecules. Glycobiology 14:53R-62R

Shim E, Shim J, Klochkova TA, Han JW, Hoon G (2012) Purification of a sex-specific lectin involved in gamete binding of Algaothamnion callophyllidiola (Rhodophyta). J Phycol 48:916-924

Shiomi K, Kamiya H, Shimizu Y (1979) Purification and characterization of an agglutinin in the red alga Agardhiella tenera. Biochim Biophys Acta 576:118-127

Shiomi K, Yamanaka H, Kikuchi T (1981) Purification and physiochemical properties of a hemagglutinin (GVA-1) in the red alga Gracilaria verrucosa. Nippon Suisan Gakkaishi 47:1079-1084

Shiomi K, Yamanaka H, Takeaki K (1980) Biochemical properties of hemagglutinins in the red alga Serraticardia maxima. Bull J Soc Sci Fish 46:1369-1373

Silva LMCM, Lima V, Holanda ML, Pinheiro PG, Rodrigues JAG, Lima MEP, Benevides NMB (2010) Antinociceptive and antiinflammatory activities of lectin from marine red alga Pterocladiella capillacea. Biol Pharm Bull 33:830-835

Simmonds P (2013) The origin of hepatitis C virus. Curr Top Microbiol Immunol 369:1-15

Singh RS, Bhari R, Kaur HP (2010) Mushroom lectins: current status and future perspectives. Crit Rev Biotechnol 30:99-126

Singh RS, Bhari R, Kaur HP (2011) Current trends of lectins from microfungi. Crit Rev Biotechnol 31:193-210

Singh RS, Kaur HP, Kanwar J (2016) Mushroom lectins as promising anticancer substances. Curr Protein Pept Sci 17:797-807

Singh RS, Thakur SR, Bansal P (2015) Algal lectins as promising biomolecules for biomedical research. Crit Rev Microbiol 41:77-88

Singh RS, Tiwary AK, Kennedy JF (1999) Lectins: sources, activities and applications. Crit Rev Biotechnol 19:145-178

Singh RS, Walia AK, Khattar JS, Singh DP, Kennedy JF (2017) Cyanobacterial lectins characteristics and their role as antiviral agents. Int J Biol Macromol 102:475-496

Souza BWS, Andrade FK, Texeira DIA, Mansilla A, Freitas ALP (2010) Haemagglutinin of the Antartic seaweed Georgiella confluens (Reinsch) Kylin: isolation and partial characterization. Polar Biol 33:1311-1318 
Stastny JJ, Gupta TK (1994) Isolation and analysis of lectin-reactive sarcoma-associated membrane glycoproteins. Anticancer Res 14: $587-591$

Sugahara T, Ohama Y, Fukuda A, Hayashi M, Kawakubo A, Kato K (2001) The cytotoxic effect of Eucheuma serra agglutinin (ESA) on cancer cells and its applications to molecular probe for drug delivery system using lipid vescicles. Cytotechnology 36:93-99

Sumiyoshi H, Mori C, Fuke I, Morita K, Kuhara S, Kondou J, Kikuchi Y, Nagamatu H, Igarashi A (1987) Complete nucleotide sequence of the Japanese encephalitis virus genome. RNA Virol 161:497-510

Takebe Y, Saucedo CJ, Lund G, Uenishi R, Hase S, Tsuchiura T, Kneteman N, Ramessar K, Tyrrell DL, Shirakura M, Wakita T, McMahon JB, O'Keefe BR (2013) Antiviral lectins from red and blue-green algae show potent in vitro and in vivo activity against hepatitis $\mathrm{C}$ virus. PLoS One 8:e64449

Teixeira EH, Arruda FVS, da Silva BR, do Nascimento KS, Carneiro VA, Cavada BS, Nagano CS, Sampaio AH (2012) Biological applications of plants and algae lectins: An overview. In: Chang CF (ed) Carbohydrates - comprehensive studies on glycobiology and glycotechnology. In Tech, Croatia, pp 533-558

Teixeira EH, Napimoga MH, Carneiro VA, de Oliveira TM, Nascimento KS, Nagano CS, Souza JB, Havt A, Pinto VPT, Goncalves RB, Farias WRL, Saker-Sampaio S, Sampaio AH, Cavada BS (2007) In vitro inhibition of oral streptococci binding to the acquired pellicle by algal lectins. J Appl Microbiol 103:1001-1006

Teixeira EH, Napimoga MH, Carneiro VA, Oliveira TM, Havt A, Martins JL, Pinto VPT, Goncalves RB, Cavada BS (2006) In vitro inhibition of streptococci binding to enamel acquired pellicle by plant lectins. J Appl Microbiol 101:111-116

Vasconcelos MA, Arruda FVS, Carneiro VA, Silva HC, Nascimento KS, Sampaio AH, Cavada B, Teixeira EH, Henriques M, Pereiro MO (2014) Effect of algae and plant lectins on planktonic growth \& biofilm formation in clinically relevant bacteria and yeasts. Biomed Res Int 2014:365272
Vieyres G, Thomas X, Descamps V, Duverlie G, Patel AH, Dubuisson J (2010) Characterization of the envelope glycoproteins associated with infectious hepatitis C virus. J Virol 84:10159-10168

Xue J, Gao Y, Horrelbeke B, Kagiampakis I, Zhao B, Demeler B, Balzarini J, Liwang PJ (2012) The role of individual charbohydrate binding sites in the function of the potent anti-HIV lectin griffithsin. Mol Pharm 9:2613-2625

Xue J, Hoorelbeke B, Kagiampakis I, Demeler B, Balzarini J, Liwang PJ (2013) The griffithsin dimer is required for high-potency inhibition of HIV-1: evidence for the manipulation of the structure of gp 120 as part of the griffithsin dimer mechanism. Antimicrob Agents Chemother 57:3976-3989

Yin A, Margolis HC, Yao Y, Grogan J, Oppenheim FG (2005) Multicomponent adsorption model for pellicle formation: the influence of salivary proteins and nonsalivary phosphoproteins on the binding of histatin 5 onto hydroxyapatite. Arch Oral Biol 51:102-110

Yoshima H, Furthmayr H, Kobata A (1980) Structures of the asparaginelinked sugar chains of glycophorin A. J Biol Chem 255:9713-9718

Zaki AM, van Boheemen S, Bestebroer TM, Osterhaus ADME, Fouchier RAM (2012) Isolation of a novel coronavirus from a man with pneumonia in Saudi Arabia. New Engl J Med 367:1814-1820

Zheng Y, Lai-Sheng L (2002) Screening of agglutinins in marine algae from Fujian coast of China. Chin J Oceanol Limnol 20:256-260

Ziolkowska NE, Keefe BR, Mori T, Zhu C, Giomarelli B, Vojdani F, Palmer KE, McMhon J, Wlodawer A (2006) Domain-swapped structure of the potent antiviral protein griffithsin and its mode of carbohydrate binding. Structure 14:1127-1135

Ziolkowska NE, Shenoy SR, O'Keefe BR, McMohan JB, Palmer KE, Dwek RA, Wormald MR, Wlodawer A (2007b) Crystallographic, thermodyanamic, and molecular modelling studies of the mode of binding of oligosaccharides to the potent antiviral protein griffithsin. Proteins: Struct Funct Bioinf 67:661-670

Ziolkowska NE, Shenoy SR, O'keefe BR, Wlodawer A (2007a) Crystallographic studies of the complexes of antiviral protein griffithsin with glucose and N-acetylglucosamine. Prot Sci 16:1485-1489 